(n)

\title{
On nonlinear coherent states properties for electron-phonon dynamics
}

Isiaka Aremua, Mahouton Norbert Hounkonnou, Ezinvi Baloïtcha

To cite this article: Isiaka Aremua, Mahouton Norbert Hounkonnou, Ezinvi Baloïtcha (2014) On nonlinear coherent states properties for electron-phonon dynamics, Journal of Nonlinear Mathematical Physics 21:1, 89-119, DOI:

https://doi.org/10.1080/14029251.2014.894723

To link to this article: https://doi.org/10.1080/14029251.2014.894723

Published online: 04 January 2021 


\title{
On nonlinear coherent states properties for electron-phonon dynamics
}

\author{
Isiaka Aremua ${ }^{\dagger}$, \\ Mahouton Norbert Hounkonnou ${ }^{\ddagger}$ \\ and Ezinvi Baloïtcha* \\ University of Abomey-Calavi, International Chair in Mathematical Physics and Applications (ICMPA-UNESCO Chair), \\ 072 B.P. 50 Cotonou, Republic of Benin \\ †claudisak@yahoo.fr. \\ ¥norbert.hounkonnou@cipma.uac.bj, \\ *ezinvi.baloitcha@cipma.uac.bj
}

Received 12 September 2013

Accepted 2 December 2013

\begin{abstract}
This work addresses a construction of a dual pair of nonlinear coherent states (NCS) in the context of changes of bases in the underlying Hilbert space for a model pertaining to an electron-phonon model in the condensed matter physics, obeying a $f$-deformed Heisenberg algebra. The existence and properties of reproducing kernel in the NCS Hilbert space are studied and discussed; the probability density and its dynamics in the basis of constructed coherent states are provided. A Glauber-Sudarshan $P$-representation of the density matrix and relevant issues related to the reproducing kernel properties are presented. Moreover, a NCS quantization of classical phase space observables is performed and illustrated in a concrete example of $q$-deformed coherent states. Finally, an exposition of quantum optical properties is given.
\end{abstract}

Keywords: Deformed Heisenberg algebra; Electron-phonon dynamics; Coherent states; Coherent state quantization.

\section{Introduction}

Coherent states (CS), known as the closest states to classical behavior of a system, play an important role in theoretical and experimental physics. First introduced as venerable objects by Schrödinger [29] in 1926 for the harmonic oscillator potential, they are since at the core of research directions in quantum optics, which is an ideal testing ground for ideas of quantum theory. Much work has been done through their theoretical generalizations including their experimental generations and applications. Then, the generalization based on group symmetry approach has led to define CS for arbitrary Lie algebras such as $s u(1,1), s u(2)$ which have found numerous applications in quantum optics [26], [34].

Further generalizations also extend to so-called nonlinear CS (NCS) or $f$-CS [20] induced by nonlinear algebras referring to $f$-deformed oscillator algebras initially introduced by Jannussis $e t$ al. [16] and Man'ko et al. [20], and spanned by the ordinary Fock-Heisenberg generators $\left\{a, a^{\dagger}, N\right\}$ coupled to a free continuous regular function $f$ of the number operator $N$, such that

$$
\begin{aligned}
A^{-} & =a f(N), \quad A^{\dagger}=f(N) a^{\dagger} \\
N & =A^{\dagger} A^{-}=N f^{2}(N), \quad\left[A^{-}, A^{\dagger}\right]=\{N+1\}-\{N\} .
\end{aligned}
$$

The $f$-deformed quantum algebras offer the advantage to be well represented in the ordinary FockHilbert space. Although they are not merely mathematical objects, NCS are useful to analyse many 
quantum mechanical systems. For instance, in [21], it has been shown that they are important in the description of the motion of a trapped ion and have some "non-classical properties" such as squeezing, amplitude-squared squeezing, antibuching, sub-Poissonian behavior.

NCS construction uses an operator similar to the displacement operator, generally denoted by $D(z), z \in \mathbb{C}$ with [28]

$$
D(z)=e^{z a^{\dagger}-\bar{z} a}, \quad\left[a, a^{\dagger}\right]=\mathbb{I}
$$

such that the CS parametrized by $z$ are given by the action of $D(z)$ on the ground state $|0\rangle$ :

$$
|z\rangle=D(z)|0\rangle
$$

This is in connection with the method proposed in [3] to define NCS by changing bases in the underlying Hilbert space, involving an interesting duality between pairs of constructed generalized CS.

Another class of CS describing quantum optical models encompasses vector coherent states (VCS) ( [8], [11], [2]), used for example in the study of spectra of two-level atomic systems placed in electromagnetic fields like the Jaynes-Cummings model [17]. For more details, see [7] (and references therein) presenting a formulation of VCS for nonlinear spin-orbit Hamiltonian model in terms of the matrix eigenvalue problem for generalized annihilation operators. A formalism of VCS construction for a system of $M$ Fermi-type modes associated with $N$ bosonic modes [22]- [33] was also given in [4]. The defined VCS satisfy, in this context, required mathematical properties of continuity, resolution of identity, temporal stability and action identity.

This work, generalizing previous investigations (see [4] and references therein), develops a construction of a dual pair of NCS, based on a $f$-deformed algebra, to describe such phenomena like the electron-phonon dynamics in condensed matter physics.

The paper is organized as follows. In Section 2, the physical model and the formalism of nonlinear coherent states (NCS) construction are described. Section 3 deals with the probability density and its dynamics in the basis of constructed coherent states. The existence and properties of reproducing kernel in the NCS Hilbert space are studied and discussed in Section 4. In Section 5, the Glauber-Sudarshan $P$-representation of the density matrix is elaborated in both the NCS and nonlinear VCS (NVCS); the associated reproducing kernel properties are studied. Section 6 presents the NCS quantization of the complex plane. In Section 7, relevant quantum optical properties are analyzed. The last section is devoted to concluding remarks.

\section{CS for electron-phonon dynamics}

In this section, we construct the CS for a physical model describing the electron-phonon dynamics given by the following Hamiltonian (with $\hbar=1$ ) [22]- [4]:

$$
H=\sum_{i=1}^{N_{B}} \omega_{i} a_{i}^{\dagger} a_{i}+\sum_{j=1}^{M} \varepsilon_{j} c_{j}^{\dagger} c_{j}+\sum_{i=1}^{N_{B}} \sum_{l=1}^{M} g_{l} c_{l}^{\dagger} c_{l}\left(a_{i}^{\dagger}+a_{i}\right)
$$

where the following commutation rules hold:

$$
\begin{gathered}
{\left[a_{i}, a_{k}^{\dagger}\right]=\delta_{i k} \mathbb{I} \quad\left\{c_{j}^{\dagger}, c_{l}\right\}=\delta_{j l} \mathbb{I}} \\
\text { Co-published by Atlantis Press and Taylor \& Francis } \\
\text { Copyright: the authors }
\end{gathered}
$$




$$
\left[a_{i}, a_{k}\right]=0 \quad\left[a_{i}^{\dagger}, a_{k}^{\dagger}\right]=0 \quad\left\{c_{j}, c_{l}\right\}=0 \quad\left\{c_{j}^{\dagger}, c_{l}^{\dagger}\right\}=0
$$

with $1 \leq i, k \leq N_{B}, 1 \leq j, l \leq M$. The set $\left\{a_{i}, a_{i}^{\dagger}, \mathbb{I}\right\}$ for each $i\left(1 \leq i \leq N_{B}\right)$ spans the ordinary Fock-Heisenberg oscillator algebra. A similar model, describing an interaction between a single mode, $\left(a, a^{\dagger}\right)$, of the radiation field with two Fermi type modes, was also investigated by Simon and Geller who outlined some physical aspects of the ensemble-averaged excited-state population dynamics [30] and showed its relevance in the study of electron-phonon dynamics in an ensemble of nearly isolated nanoparticles, in the context of quantum effects in condensed matter systems. The vibrational spectrum of a nanoparticle is here provided by the localized electronic impurity states in doped nanocrystal $[22,33]$. The impurity states are used to probe the energy relaxation by phonon emission.

The deformed version of the Hamiltonian (2.1) is obtained by performing the correspondences

$$
a_{l} \rightarrow a_{l} f_{l}\left(N_{l}\right) \quad a_{l}^{\dagger} \rightarrow f_{l}\left(N_{l}\right) a_{l}^{\dagger} \quad 1 \leq l \leq N_{B}
$$

with the following nonlinear commutation rules for the bosonic operators:

$$
\begin{array}{r}
{\left[N_{l}, a_{l} f_{l}\left(N_{l}\right)\right]=-a_{l} f_{l}\left(N_{l}\right), \quad\left[N_{l}, f_{l}\left(N_{l}\right) a_{l}^{\dagger}\right]=f_{l}\left(N_{l}\right) a_{l}^{\dagger},} \\
{\left[a_{l} f_{l}\left(N_{l}\right), f_{l}\left(N_{l}\right) a_{l}^{\dagger}\right]=\left(N_{l}+1\right) f_{l}^{2}\left(N_{l}+1\right)-N_{l} f_{l}^{2}\left(N_{l}\right),}
\end{array}
$$

$f_{l}\left(N_{l}\right)$ being a reasonably well behaved real function of the number operator $N_{l}$. If $f_{l}\left(N_{l}\right)=1$, then the nonlinear algebra in (2.4) reduces to the ordinary non-deformed oscillator algebra.

As a matter of obtaining the eigenvalues and eigenvectors for our system, the Hamiltonian (2.1) can be conveniently diagonalized by introducing the following self adjoint operator:

$$
B_{[\mathbf{k}]_{l}}=\omega_{l} A_{[\mathbf{k}]_{l}}^{\dagger} A_{[\mathbf{k}]_{l}}+\frac{\varepsilon_{[\mathbf{k}]}}{N_{B}}-\frac{g_{[\mathbf{k}]}^{2}}{\omega_{l}} \quad \text { with } \quad A_{[\mathbf{k}]_{l}}=a_{l} f_{l}\left(N_{l}\right)+\frac{g_{[\mathbf{k}]}}{\omega_{l}}
$$

where $g_{[\mathbf{k}]}, \varepsilon_{[\mathbf{k}]}$ are defined as [4]

$$
\begin{gathered}
g_{[\mathbf{k}]}:=\sum_{k_{j} \in[\mathbf{k}]} k_{j} g_{j} \quad \varepsilon_{[\mathbf{k}]}:=\sum_{k_{j} \in[\mathbf{k}]} k_{j} \varepsilon_{j} \quad 1 \leq j \leq M \quad[\mathbf{k}] \in \Gamma, \\
\Gamma=\{(0,0, \cdots, 0,0),(1,0, \cdots, 0,0), \ldots,(1,1 \cdots, 1,1)\} .
\end{gathered}
$$

Then, using the relations (2.5)-(2.7) leads to the reduced Hamiltonian in the form:

$$
B_{[\mathbf{k}][\mathbf{n}]}=\sum_{l=1}^{N_{B}} \omega_{l} A_{[\mathbf{k}]_{l}}^{\dagger} A_{[\mathbf{k}]_{l}}+\varepsilon_{[\mathbf{k}]}-g_{[\mathbf{k}]}^{2} \sum_{l=1}^{N_{B}} \frac{1}{\omega_{l}} \quad \text { with } \quad[\mathbf{n}]:=n_{1}, n_{2}, \ldots, n_{N_{B}} .
$$

In the next subsection, we define the operators useful for our construction by transforming CS into their deformed correspondents with an operator $T$, and conversely into their duals with the inverse operator $T^{-1}$, such that $T T^{-1}=\mathbb{I}$, the identity operator of the considered Hilbert space. 


\subsection{Rescaled basis states, eigenvalues and eigenvectors}

For all $l, 1 \leq l \leq N_{B}, \mathfrak{H}_{l}$ denotes the separable Hilbert space spanned by the eigenvectors $\phi_{n_{l}}, n_{l}=$ $0,1,2, \ldots, \infty$ of the number operator $N_{l}$.

On $\mathfrak{H}_{l}$, let $T$ be an operator densely defined and closed in the domain denoted by $\mathscr{D}(T)$. Suppose that $T^{-1}$ exists and is densely defined with domain $\mathscr{D}\left(T^{-1}\right)$. Moreover, the vectors $\phi_{n_{l}} \in \mathscr{D}(T) \cap$ $\mathscr{D}\left(T^{-1}\right)$ for all $n_{l}$ and there exist non-empty open sets $\mathscr{D}_{T}$ and $\mathscr{D}_{T^{-1}}$ in $\mathbb{C}$ such that $\eta_{z_{l}} \in \mathscr{D}(T), \forall z_{l} \in$ $\mathscr{D}_{T}$ and $\eta_{z_{l}} \in \mathscr{D}\left(T^{-1}\right), \forall z_{l} \in \mathscr{D}_{T^{-1}}$.

The operator $T$ being densely defined and closed, then the operator $\mathscr{S}$ such that $\mathscr{S}=T^{*} T$, with domain $\mathscr{D}(\mathscr{S})$, where $T^{*}$ is the operator adjoint of $T$, is self adjoint.

Let $\mathfrak{H}_{l}^{\mathscr{S}}$ be the completion of $\mathscr{D}(T)$ in the scalar product

$$
\left\langle\phi_{n_{l}} \mid \psi_{n_{l}}\right\rangle_{\mathfrak{H}_{l}^{\mathscr{S}}}=\left\langle\phi_{n_{l}} \mid T^{*} T \psi_{n_{l}}\right\rangle_{\mathfrak{H}_{l}}=\left\langle\phi_{n_{l}} \mid \mathscr{S} \psi_{n_{l}}\right\rangle_{\mathfrak{H}_{l}}, \quad\left|\psi_{n_{l}}\right\rangle \in \mathscr{D}(\mathscr{S}),
$$

where $\mathfrak{H}_{l}^{\mathscr{S}}$ is spanned by the vectors

$$
\left|\phi_{n_{l}}^{\mathscr{S}}\right\rangle=T^{-1}\left|\phi_{n_{l}}\right\rangle, \quad\left|\phi_{n_{l}}\right\rangle \in \mathscr{D}\left(T^{-1}\right),
$$

and $\mathfrak{H}_{l}^{\mathscr{S}^{-1}}$ the completion of $\mathscr{D}\left(T^{*-1}\right)$ in the scalar product

$$
\left\langle\phi_{n_{l}} \mid \psi_{n_{l}}\right\rangle_{\mathfrak{H}_{l}^{S^{-1}}}=\left\langle\phi_{n_{l}} \mid T^{-1} T^{*-1} \psi_{n_{l}}\right\rangle_{\mathfrak{H}_{l}}=\left\langle\phi_{n_{l}} \mid \mathscr{S}^{-1} \psi_{n_{l}}\right\rangle_{\mathfrak{H}_{l}}, \quad\left|\psi_{n_{l}}\right\rangle \in \mathscr{D}\left(\mathscr{S}^{-1}\right),
$$

where $\mathscr{D}\left(\mathscr{S}^{-1}\right)$ is the domain of $\mathscr{S}^{-1}$, with $\mathfrak{H}_{l}^{\mathscr{S}^{-1}}$ spanned by the vectors

$$
\left|\phi_{n_{l}}^{\mathscr{S}^{-1}}\right\rangle=T\left|\phi_{n_{l}}\right\rangle, \quad\left|\phi_{n_{l}}\right\rangle \in \mathscr{D}(T) .
$$

Moreover, if the spectrum of $\mathscr{S}$ is bounded away from zero, then $\mathscr{S}^{-1}$ is bounded and there follow the inclusions

$$
\mathfrak{H}_{l}^{\mathscr{S}} \subset \mathfrak{H}_{l} \subset \mathfrak{H}_{l}^{\mathscr{S}^{-1}} .
$$

The so-defined Hilbert spaces $\mathfrak{H}_{l}^{\mathscr{S}}, \mathfrak{H}_{l}$ and $\mathfrak{H}_{l}^{\mathscr{S}^{-1}}$ are called a Gelfand triple.

On $\mathfrak{H}_{l}^{\mathscr{S}}$ the transformed counterparts of the operators $a_{l}, a_{l}^{\dagger}, N_{l}$ defined on $\mathfrak{H}_{l}$ are [3]

$$
a_{l}^{\mathscr{S}}=T^{-1} a_{l} T, \quad a_{l}^{\mathscr{S}^{\dagger}}=T^{-1} a_{l}^{\dagger} T, \quad N_{l}^{\mathscr{S}}=T^{-1} N_{l} T,
$$

and their actions on $\mathfrak{H}_{l}^{\mathscr{S}}$ defined by

$$
a_{l}^{\mathscr{S}}\left|\phi_{n_{l}}^{\mathscr{S}}\right\rangle:=\sqrt{n_{l}}\left|\phi_{n_{l}-1}^{\mathscr{S}}\right\rangle, \quad a_{l}^{\mathscr{S} \dagger}\left|\phi_{n_{l}}^{\mathscr{S}}\right\rangle:=\sqrt{n_{l}+1}\left|\phi_{n_{l}+1}^{\mathscr{S}}\right\rangle, \quad N_{l}^{\mathscr{S}}\left|\phi_{n_{l}}^{\mathscr{S}}\right\rangle:=n_{l}\left|\phi_{n_{l}}^{\mathscr{S}}\right\rangle .
$$

These operators satisfy the same commutation relations as $a_{l}, a_{l}^{\dagger}$ and $N_{l}$ :

$$
\left[a_{l}^{\mathscr{S}}, a_{l}^{\mathscr{S}^{\dagger}}\right]=\mathbb{I}, \quad\left[a_{l}^{\mathscr{S}}, N_{l}^{\mathscr{S}}\right]=a_{l}^{\mathscr{S}}, \quad\left[a_{l}^{\mathscr{S} \dagger}, N_{l}^{\mathscr{S}}\right]=-a_{l}^{\mathscr{S} \dagger} .
$$

The transformed operators $a_{l}^{\mathscr{S}^{-1}}, a_{l}^{\mathscr{S}^{-1} \dagger}, N_{l}^{\mathscr{S}^{-1}}$ of the operators $a_{l}, a_{l}^{\dagger}, N_{l}$ defined on $\mathfrak{H}_{l}$, are characterized as follows:

$$
a_{l}^{\mathscr{S}^{-1}}=T a_{l} T^{-1}, \quad a_{l}^{\mathscr{S}^{-1} \dagger}=T a_{l}^{\dagger} T^{-1}, \quad N_{l}^{\mathscr{S}^{-1}}=T N_{l} T^{-1}
$$

acting on $\mathfrak{H}_{l}^{\mathscr{S}^{-1}}$ in a similar manner as in (2.15) and satisfying the commutation relations

$$
\left[a_{l}^{\mathscr{S}^{-1}}, a_{l}^{\mathscr{S}^{-1} \dagger}\right]=\mathbb{I}, \quad\left[a_{l}^{\mathscr{S}^{-1}}, N_{l}^{\mathscr{S}^{-1}}\right]=a_{l}^{\mathscr{S}^{-1}}, \quad\left[a_{l}^{\mathscr{S}^{-1}}{ }^{\dagger}, N_{l}^{\mathscr{S}^{-1}}\right]=-a_{l}^{\mathscr{S}^{-1} \dagger} .
$$


The operators $T, T^{-1}, \mathscr{S}$ can be written as follows:

$$
\begin{aligned}
T & :=T\left(N_{l}\right)=\sum_{n_{l}=0}^{\infty} t\left(n_{l}\right)\left|\phi_{n_{l}}\right\rangle\left\langle\phi_{n_{l}}\left|, \quad T^{-1}:=T\left(N_{l}\right)^{-1}=\sum_{n_{l}=0}^{\infty} \frac{1}{t\left(n_{l}\right)}\right| \phi_{n_{l}}\right\rangle\left\langle\phi_{n_{l}}\right|, \\
\mathscr{S} & :=\mathscr{S}\left(N_{l}\right)=\sum_{n_{l}=0}^{\infty} t\left(n_{l}\right)^{2}\left|\phi_{n_{l}}\right\rangle\left\langle\phi_{n_{l}}\right| .
\end{aligned}
$$

For all $l$, the action of $f_{l}\left(N_{l}\right)$ on the basis vectors $\left|\phi_{n_{l}}\right\rangle$ of the separable Hilbert space $\mathfrak{H}_{l}$ can be also defined as:

$$
f_{l}\left(N_{l}\right)\left|\phi_{n_{l}}\right\rangle:=\frac{t\left(n_{l}\right)}{t\left(n_{l}-1\right)}\left|\phi_{n_{l}}\right\rangle=f_{l}\left(n_{l}\right)\left|\phi_{n_{l}}\right\rangle
$$

where

$$
t\left(n_{l}\right)=f_{l}\left(n_{l}\right) f_{l}\left(n_{l}-1\right) \cdots f(1):=f_{l}\left(n_{l}\right) !
$$

are real numbers, possessing the properties:

- $t(0)=1$ and $t\left(n_{l}\right)=t\left(n_{l}^{\prime}\right)$ if and only if $n_{l}=n_{l}^{\prime}$;

- $0<t\left(n_{l}\right)<\infty$;

- the Cauchy criterion implies that the finiteness condition for the limit

$$
\lim _{n_{l} \rightarrow \infty}\left[\frac{t\left(n_{l}\right)}{t\left(n_{l}+1\right)}\right]^{2} \frac{1}{n_{l}+1}=\rho<\infty
$$

holds. This condition implies that the series $S\left(r^{2}\right):=\sum_{n_{l}=0}^{\infty} \frac{r^{2 n_{l}}}{\left[t\left(n_{l}\right)\right]^{2} n_{l} !}$ converges for all $r<$ $L=1 / \sqrt{\rho}$.

For all $l$, we define the function of the operator $N_{l}, f_{l}\left(N_{l}\right)^{-1}$ acting on the basis vectors $\left|\phi_{n_{l}}\right\rangle$ of $\mathfrak{H}_{l}$ in the following manner:

$$
f_{l}\left(N_{l}\right)^{-1}\left|\phi_{n_{l}}\right\rangle:=\frac{t\left(n_{l}-1\right)}{t\left(n_{l}\right)}\left|\phi_{n_{l}}\right\rangle=\frac{1}{f_{l}\left(n_{l}\right)}\left|\phi_{n_{l}}\right\rangle
$$

The real numbers $t\left(n_{l}\right)$ satisfying the above two first properties are such that the finiteness condition for the limit

$$
\lim _{n_{l} \rightarrow \infty}\left[\frac{t\left(n_{l}+1\right)}{t\left(n_{l}\right)}\right]^{2} \frac{1}{n_{l}+1}=\tilde{\rho}<\infty
$$

implies that the series $S^{\prime}\left(r^{2}\right):=\sum_{n_{l}=0}^{\infty} \frac{r^{2 n_{l}}\left(t\left(n_{l}\right)\right)^{2}}{n_{l} !}$ converges for all $r<\tilde{L}=1 / \sqrt{\tilde{\rho}}$. Since $\left|\phi_{n_{l}}^{\mathscr{S}^{-1}}\right\rangle=$ $T\left|\phi_{n_{l}}\right\rangle$, then

$$
\left|\phi_{n_{l}}^{\mathscr{S}^{-1}}\right\rangle=t\left(n_{l}\right)\left|\phi_{n_{l}}\right\rangle=f_{l}\left(n_{l}\right) !\left|\phi_{n_{l}}\right\rangle .
$$

As matter of clarity, recall $\mathfrak{H}_{l}^{\mathscr{S}} \subset \mathfrak{H}_{l} \subset \mathfrak{H}_{l}^{\mathscr{S}^{-1}}$ and the operators $a_{l}^{\mathscr{S}}$ and $a_{l}^{\mathscr{S}^{-1}}$ act in $\mathfrak{H}_{l}^{\mathscr{S}}$ and $\mathfrak{H}_{l}^{\mathscr{S}^{-1}}$, respectively. To avoid any confusion in what follows, we denote $A_{l}=a_{l}^{\mathscr{S}}$ when $a_{l}^{\mathscr{S}}$ acts on $\mathfrak{H}_{l}$, i.e. prolongating the action of $a_{l}^{\mathscr{S}}$ into $\mathfrak{H}_{l}$, and $A_{l}^{\prime}=\left.a_{l}^{\mathscr{S}^{-1}}\right|_{\mathfrak{H}_{l}}$ restricting the action of $a_{l}^{\mathscr{S}^{-1}}$ to $\mathfrak{H}_{l}$. 
Since $T, T^{-1}$ and $\mathscr{S}$ are positive operators, the adjoints of $A_{l}$ and $A_{l}^{\prime}$ on $\mathfrak{H}_{l}$ take the form:

$$
A_{l}^{\dagger}=T a_{l}^{\dagger} T^{-1}, \quad A_{l}^{\prime \dagger}=T^{-1} a_{l}^{\dagger} T
$$

From the relations

$$
f_{l}\left(N_{l}\right)\left|\phi_{n_{l}}\right\rangle=f_{l}\left(n_{l}\right)\left|\phi_{n_{l}}\right\rangle,\left|\phi_{n_{l}}^{\mathscr{S}}\right\rangle=\frac{1}{f_{l}\left(n_{l}\right) !}\left|\phi_{n_{l}}\right\rangle
$$

and

$$
f_{l}\left(N_{l}\right)^{-1}\left|\phi_{n_{l}}\right\rangle=\frac{1}{f_{l}\left(n_{l}\right)}\left|\phi_{n_{l}}\right\rangle,\left|\phi_{n_{l}}^{\mathscr{S}^{-1}}\right\rangle=f_{l}\left(n_{l}\right) !\left|\phi_{n_{l}}\right\rangle,
$$

we infer their actions as

$$
A_{l}\left|\phi_{n_{l}}\right\rangle=\sqrt{n_{l}} f_{l}\left(n_{l}\right)\left|\phi_{n_{l}-1}\right\rangle, \quad A_{l}^{\dagger}\left|\phi_{n_{l}}\right\rangle=\sqrt{n_{l}+1} f_{l}\left(n_{l}+1\right)\left|\phi_{n_{l}+1}\right\rangle .
$$

The vectors $\left|\phi_{n_{l}}^{\mathscr{S}}\right\rangle$ and $\left|\phi_{n_{l}}^{\mathscr{S}^{-1}}\right\rangle$, defined in (2.27) and (2.28), are called rescaled basis states [3].

Then we get

$$
A_{l}=a_{l} f_{l}\left(N_{l}\right), A_{l}^{\dagger}=f_{l}\left(N_{l}\right) a_{l}^{\dagger}, A_{l}^{\prime}=a_{l} f_{l}\left(N_{l}\right)^{-1}, A_{l}^{\prime \dagger}=f_{l}\left(N_{l}\right)^{-1} a_{l}^{\dagger}
$$

with the following relations

$$
\left[A_{l}, A_{l}^{\prime \dagger}\right]=\mathbb{I} \quad\left[A_{l}^{\prime}, A_{l}^{\dagger}\right]=\mathbb{I} \quad 1 \leq l \leq N_{B} .
$$

From the relations (2.29), the operators $a_{l}^{\mathscr{S}}, a_{l}^{\mathscr{S}^{-1} \dagger}$ and $N_{l}$ can be expressed as

$$
\begin{aligned}
& A_{l}=\sum_{n_{l}=0}^{\infty} \sqrt{n_{l}} f_{l}\left(n_{l}\right)\left|\phi_{n_{l}-1}\right\rangle\left\langle\phi_{n_{l}}\left|, \quad A_{l}^{\dagger}=\sum_{n_{l}=0}^{\infty} \sqrt{n_{l}} f\left(n_{l}\right)\right| \phi_{n_{l}}\right\rangle\left\langle\phi_{n_{l}-1}\right| \\
& N_{l}=\sum_{n_{l}=0}^{\infty} n_{l}\left|\phi_{n_{l}}\right\rangle\left\langle\phi_{n_{l}}\right| .
\end{aligned}
$$

For all $l$, we define, on the Hilbert space $\mathfrak{H}_{l}$, the momentum operator $P_{l}$ as

$$
P_{l}:=\frac{a_{l} f_{l}\left(N_{l}\right)-f_{l}\left(N_{l}\right)^{-1} a_{l}^{\dagger}}{i \sqrt{2}} \quad \text { with } \quad\left[a_{l} f_{l}\left(N_{l}\right), \sqrt{2} P_{l}\right]=i \mathbb{I}
$$

The eigenvectors of $B_{[\mathbf{k}]_{l}}$ are given, with $\left|\Phi_{0_{l}}^{[\mathbf{k}]}\right\rangle=e^{i \sqrt{2} \frac{g}{\omega_{l}} P_{l}}|0\rangle$, by

$$
\left|\Phi_{n_{l}}^{[\mathbf{k}]}\right\rangle=e^{i \sqrt{2} \frac{g}{\left.\omega_{l} \mathbf{k}\right]} P_{l}}\left|\phi_{n_{l}}\right\rangle=\frac{\left(A_{[\mathbf{k}] l}^{\dagger}\right)^{n_{l}}}{\sqrt{n_{l} !} f_{l}\left(n_{l}\right) !}\left|\Phi_{0_{l}}^{[\mathbf{k}]}\right\rangle, \quad A_{[\mathbf{k}]_{l}}=e^{i \sqrt{2} \frac{g[\mathbf{k}]}{\omega_{l}} P_{l}} a_{l} f_{l}\left(N_{l}\right) e^{-i \sqrt{2} \frac{g}{\left.g_{l}\right]} P_{l}}
$$

while for the operators $B_{[\mathbf{k}]}$ the eigenvalues $E_{\mathbf{n}}^{[\mathbf{k}]}$ and eigenfunctions $\left|\Phi_{\mathbf{n}}^{[\mathbf{k}]}\right\rangle$ are computed as follows:

$$
E_{\mathbf{n}}^{[\mathbf{k}]}=\sum_{l=1}^{N_{B}} \omega_{l} n_{l} f_{l}^{2}\left(n_{l}\right)+\varepsilon_{[\mathbf{k}]}-g_{[\mathbf{k}]}^{2} \sum_{l=1}^{N_{B}} \frac{1}{\omega_{l}}, \quad\left|\Phi_{\mathbf{n}}^{[\mathbf{k}]}\right\rangle=\bigotimes_{l=1}^{N_{B}}\left|\Phi_{n_{l}}^{[\mathbf{k}]}\right\rangle .
$$



that

Let us denote the duals of the operators $B_{[\mathbf{k}]_{l}}, A_{[\mathbf{k}]_{l}}, A_{[\mathbf{k}]_{l}}^{\dagger}$ by $B_{[\mathbf{k}]_{l}}^{\prime}, A_{[\mathbf{k}]_{l}}^{\prime}, A_{[\mathbf{k}]_{l}}^{\prime \dagger}$, respectively, such

$$
B_{[\mathbf{k}]_{l}}^{\prime}:=\omega_{l} A_{[\mathbf{k}]_{l}^{\prime}}^{\prime \dagger} A_{[\mathbf{k}]_{l}}^{\prime}+\frac{\varepsilon_{[\mathbf{k}]}}{N_{B}}-\frac{g_{[\mathbf{k}]}^{2}}{\omega_{l}}, \quad A_{[\mathbf{k}]_{l}}^{\prime}:=a_{l} f_{l}\left(N_{l}\right)^{-1}+\frac{g_{[\mathbf{k}]}}{\omega_{l}}
$$

and define, on the separable Hilbert space $\mathfrak{H}_{l}$, the dual of the momentum operator $P_{l}$ by

$$
P_{l}^{\prime}:=\frac{a_{l} f_{l}\left(N_{l}\right)^{-1}-f_{l}\left(N_{l}\right) a_{l}^{\dagger}}{i \sqrt{2}} \quad \text { with } \quad\left[a_{l} f_{l}\left(N_{l}\right)^{-1}, \sqrt{2} P_{l}^{\prime}\right]=i \mathbb{I} .
$$

Then, the eigenvectors of $B_{[\mathbf{k}]_{l}}^{\prime}$ are given, with $\left|\Phi_{0_{l}}^{\prime[\mathbf{k}]}\right\rangle=e^{i \sqrt{2} \frac{g}{\left.\sigma_{\mathbf{k}}\right]} P_{l}^{\prime}}|0\rangle$, by

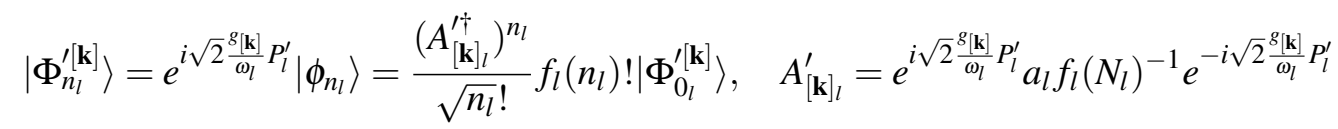

corresponding to the eigenvalues

$$
E_{\mathbf{n}}^{\prime \mathbf{k}]}=\sum_{l=1}^{N_{B}} \omega_{l} n_{l} f_{l}^{-2}\left(n_{l}\right)+\varepsilon_{[\mathbf{k}]}-g_{[\mathbf{k}]}^{2} \sum_{l=1}^{N_{B}} \frac{1}{\omega_{l}} .
$$

It then comes that the eigenvectors of $H$ associated to the eigenvalues $E_{\mathbf{n}}^{[\mathbf{k}]}$ are given by

$$
\left|\varphi_{\mathbf{n}}^{[\mathbf{k}]}\right\rangle=\left|\Phi_{\mathbf{n}}^{[\mathbf{k}]}\right\rangle \otimes\left|\Psi_{[\mathbf{k}]}\right\rangle, \quad\left|\Psi_{[\mathbf{k}]}\right\rangle=\left(c_{1}^{\dagger}\right)^{k_{1}}\left(c_{2}^{\dagger}\right)^{k_{2}} \cdots\left(c_{j}^{\dagger}\right)^{k_{j}} \cdots\left(c_{M}^{\dagger}\right)^{k_{M}}\left|\Psi_{(0,0, \ldots, 0)}\right\rangle .
$$

Since the operators $e^{i \sqrt{2} \frac{g[\mathbf{k}]}{\omega_{l}} P_{l}}, e^{i \sqrt{2} \frac{g}{\sigma_{l} \mid \mathbf{k}} P_{l}^{\prime}}$ are unitary and act on $\mathfrak{H}_{l}$, the sets $\left\{\left|\Phi_{n_{l}}^{[\mathbf{k}]}\right\rangle\right\}_{n_{l}=0}^{\infty}$ and $\left\{\left|\Phi_{n_{l}}^{\prime[\mathbf{k}]}\right\rangle\right\}_{n_{l}=0}^{\infty}$ are both basis vectors of $\mathfrak{H}_{l}$.

\subsection{Construction of the nonlinear coherent states (NCS)}

From the relations (2.34) and (2.38), we arrive at the following definitions:

$$
\left\{n_{l}\right\} !:=n_{l} !\left[f_{l}\left(n_{l}\right) !\right]^{2}, \quad\left\{n_{l}\right\}_{d} !:=\frac{n_{l} !}{\left[f_{l}\left(n_{l}\right) !\right]^{2}} .
$$

For all $l$, we consider now, on $\mathfrak{H}_{l}$, the displacement operators, related to the dual pair $\mathfrak{H}_{l}^{F}, \mathfrak{H}_{l}^{F-1}$, as:

$$
\begin{aligned}
& D_{l}\left(z_{l}\right):=e^{l \sqrt{2} \frac{\left.g^{\prime} \mathbf{k}\right]}{\omega_{l}} P_{l}} e^{z_{l} A_{l}^{\prime \dagger}-\bar{z}_{l} A_{l}}, z_{l} \in \mathscr{D}_{l}=\left\{z_{l} \in \mathbb{C} /\left|z_{l}\right|<L=1 / \sqrt{\rho}\right\}, \\
& D_{l}^{\prime}\left(z_{l}\right):=e^{l \sqrt{2} \frac{\left.g^{g} \mathbf{k}\right]}{\omega_{l}} P_{l}^{\prime}} e^{z_{l} A_{l}^{\dagger}-\bar{z}_{l} A_{l}^{\prime}}, z_{l} \in \tilde{\mathscr{D}}_{l}=\left\{z_{l} \in \mathbb{C} /\left|z_{l}\right|<\tilde{L}=1 / \sqrt{\tilde{\rho}}\right\} .
\end{aligned}
$$

Since the operators $e^{l \sqrt{2} \frac{g[\mathbf{k}]}{\omega_{l}} P_{l}}$ and $e^{l \sqrt{2} \frac{g[\mathbf{k}]}{\omega_{l}} P_{l}^{\prime}}$ are unitary on $\mathfrak{H}_{l}$, the above operators are also unitary on $\mathfrak{H}_{l}$, and, in view of the relations

$$
D_{l}\left(z_{l}\right) D_{l}\left(z_{l}^{\prime}\right)=e^{l 2 \sqrt{2} \frac{g}{\left.g_{l} \mid \mathbf{k}\right]} P_{l}} e^{l I m\left(\bar{z}_{l} z_{l}^{\prime}\right)} D_{l}\left(z_{l}+z_{l}^{\prime}\right)
$$




$$
D_{l}^{\prime}\left(z_{l}\right) D_{l}^{\prime}\left(z_{l}^{\prime}\right)=e^{l 2 \sqrt{2} \frac{g[\mathbf{k}]}{\omega_{l}} P_{l}^{\prime}} e^{I I m\left(\bar{z}_{l} z_{l}^{\prime}\right)} D_{l}^{\prime}\left(z_{l}+z_{l}^{\prime}\right)
$$

which hold if $z_{l}, z_{l}^{\prime}, z_{l}+z_{l}^{\prime} \in \mathscr{D}_{l}$ (resp. $\in \tilde{\mathscr{D}}_{l}$ ), they realize a unitary projective representation of the Weyl-Heisenberg group on $\mathfrak{H}_{l}$.

By using the Baker-Campbell-Hausdorff identity, we obtain

$$
\begin{aligned}
& D_{l}\left(z_{l}\right)=e^{l \sqrt{2} \frac{g^{g}|\mathbf{k}|}{\omega_{l}} P_{l}} e^{-\left.\frac{1}{2}|z|^{2}\right|^{2} e^{z_{l} A_{l}^{\prime \dagger}} e^{-\bar{z}_{l} A_{l}},} \\
& D_{l}^{\prime}\left(z_{l}\right)=e^{l \sqrt{2} \frac{g^{g}|\mathbf{k}|}{\omega_{l}} P_{l}^{\prime}} e^{-\frac{1}{2}\left|z_{l}\right|^{2}} e^{z_{l} A_{l}^{\dagger}} e^{-\bar{z}_{l} A_{l}^{\prime}} .
\end{aligned}
$$

From (2.45), for all $z_{l} \in \mathscr{D}_{l}$, the NCS

$$
D_{l}\left(z_{l}\right)|0\rangle_{l}=e^{-\frac{1}{2}\left|z_{l}\right|^{2}} e^{l \sqrt{2} \frac{g, \mathbf{k}]}{\omega_{l}} P_{l}} \sum_{n_{l}=0}^{\infty} \frac{z_{l}^{n_{l}}}{\sqrt{\left\{n_{l}\right\} !}}\left|\phi_{n_{l}}\right\rangle
$$

become in the domain $\mathscr{D}_{l}$ given in (2.42):

$$
D_{l}\left(z_{l}\right)|0\rangle_{l}:=\left(\mathscr{N}\left(\left|z_{l}\right|^{2}\right)\right)^{-1 / 2} \sum_{n_{l}=0}^{\infty} \frac{z_{l}^{n_{l}}}{\sqrt{\left\{n_{l}\right\} !}}\left|\Phi_{n_{l}}^{[\mathbf{k}]}\right\rangle
$$

with the normalization factor, by using (2.41), given by

$$
\mathscr{N}\left(\left|z_{l}\right|^{2}\right)=\sum_{n_{l}=0}^{\infty} \frac{\left(\bar{z}_{l} z_{l}\right)^{n_{l}}}{\left\{n_{l}\right\} !} .
$$

These vectors correspond to the well-known NCS of quantum optics [20], related to the basis vectors $\left\{\left|\Phi_{n_{l}}^{[\mathbf{k}]}\right\rangle\right\}_{n_{l}=0}^{\infty}$ of the separable Hilbert space $\mathfrak{H}_{l}$.

Similarly, from (2.46), we determine, for all $z_{l} \in \tilde{\mathscr{D}}_{l}$, the NCS

$$
D_{l}^{\prime}\left(z_{l}\right)|0\rangle_{l}=\left(\mathscr{N}^{\prime}\left(\left|z_{l}\right|^{2}\right)\right)^{-1 / 2} \sum_{n_{l}=0}^{\infty} \frac{z_{l}^{n_{l}}}{\sqrt{\left\{n_{l}\right\}_{d} !}}\left|\Phi_{n_{l}}^{\prime[\mathbf{k}]}\right\rangle
$$

with

$$
\mathscr{N}^{\prime}\left(\left|z_{l}\right|^{2}\right)=\sum_{n_{l}=0}^{\infty} \frac{\left(\bar{z}_{l} z_{l}\right)^{n_{l}}}{\left\{n_{l}\right\}_{d} !}
$$

Setting now

$$
|0\rangle=|0\rangle_{1} \otimes|0\rangle_{2} \otimes \cdots \otimes|0\rangle_{l} \cdots \otimes|0\rangle_{N_{B}}
$$

and considering the displacement operators $D_{\mathbf{n}}(\mathbf{z})$ and $D_{\mathbf{n}}^{\prime}(\mathbf{z})$ :

$$
D_{\mathbf{n}}(\mathbf{z}):=\bigotimes_{l=1}^{N_{B}} D_{l}\left(z_{l}\right), \quad D_{\mathbf{n}}^{\prime}(\mathbf{z}):=\bigotimes_{l=1}^{N_{B}} D_{l}^{\prime}\left(z_{l}\right),
$$

the action of $D_{\mathbf{n}}(\mathbf{z})$ on $|0\rangle$ can be realized as:

$$
D_{\mathbf{n}}(\mathbf{z})|0\rangle=\bigotimes_{l=1}^{N_{B}} D_{l}\left(z_{l}\right)\left(|0\rangle_{1} \otimes|0\rangle_{2} \otimes \cdots \otimes|0\rangle_{l} \otimes \cdots \otimes|0\rangle_{N_{B}}\right)
$$




$$
\begin{aligned}
= & D_{1}\left(z_{1}\right)|0\rangle_{1} \otimes D_{2}\left(z_{2}\right)|0\rangle_{2} \otimes \cdots \otimes D_{l}\left(z_{l}\right)|0\rangle_{l} \otimes \cdots \otimes D_{N_{B}}\left(z_{N_{B}}\right)|0\rangle_{N_{B}} \\
= & \left(\mathscr{N}\left(\left|z_{1}\right|^{2}\right)\right)^{-1 / 2} \sum_{n_{1}=0}^{\infty} \frac{z_{1}^{n_{1}}}{\sqrt{\left\{n_{1}\right\} !}}\left(\mathscr{N}\left(\left|z_{2}\right|^{2}\right)\right)^{-1 / 2} \sum_{n_{2}=0}^{\infty} \frac{z_{2}^{n_{2}}}{\sqrt{\left\{n_{2}\right\} !}} \\
& \cdots\left(\mathscr{N}\left(\left|z_{l}\right|^{2}\right)\right)^{-1 / 2} \sum_{n_{l}=0}^{\infty} \frac{z_{l}^{n_{l}}}{\sqrt{\left\{n_{l}\right\} !}} \cdots\left(\mathscr{N}\left(\left|z_{N_{B}}\right|^{2}\right)\right)^{-1 / 2} \sum_{n_{N_{B}}=0}^{\infty} \frac{z_{N_{B}}^{n_{N_{B}}}}{\sqrt{\left\{n_{N_{B}}\right\} !}} \\
& \times\left(\left|\Phi_{n_{1}}^{[\mathbf{k}]}\right\rangle \otimes\left|\Phi_{n_{2}}^{[\mathbf{k}]}\right\rangle \otimes \cdots \otimes\left|\Phi_{n_{l}}^{[\mathbf{k}]}\right\rangle \otimes \cdots \otimes\left|\Phi_{n_{N_{B}}}^{[\mathbf{k}]}\right\rangle\right) .
\end{aligned}
$$

Putting

$$
\mathscr{N}\left(|\mathbf{z}|^{2}\right):=\prod_{l=1}^{N_{B}} \mathscr{N}_{l}\left(\left|z_{l}\right|^{2}\right), \quad \mathbf{z}^{\mathbf{n}}:=\prod_{l=1}^{N_{B}} z_{l}^{n_{l}}
$$

and

$$
\mathbf{n} !:=n_{1} ! n_{2} ! \cdots n_{N_{B}} !, \quad f(\mathbf{n}) !:=f\left(n_{1}\right) ! f\left(n_{2}\right) ! \cdots f\left(n_{N_{B}}\right) !,
$$

we derive the NCS as follows:

$$
\left|\eta_{\mathbf{z}}\right\rangle:=D_{\mathbf{n}}(\mathbf{z})|0\rangle=\left(\mathscr{N}\left(|\mathbf{z}|^{2}\right)\right)^{-1 / 2} \sum_{\mathbf{n}=0}^{\infty} \frac{\mathbf{z}^{\mathbf{n}}}{\sqrt{\{\mathbf{n}\} !}}\left|\Phi_{\mathbf{n}}^{[\mathbf{k}]}\right\rangle, \quad \mathbf{z} \in \mathscr{D}
$$

and

$$
\left|\eta_{\mathbf{z}}^{\prime}\right\rangle:=D_{\mathbf{n}}^{\prime}(\mathbf{z})|0\rangle=\left(\mathscr{N}^{\prime}\left(|\mathbf{z}|^{2}\right)\right)^{-1 / 2} \sum_{\mathbf{n}=0}^{\infty} \frac{\mathbf{z}^{\mathbf{n}}}{\sqrt{\{\mathbf{n}\}_{d} !}}\left|\Phi_{\mathbf{n}}^{\prime[\mathbf{k}]}\right\rangle, \quad \mathbf{z} \in \tilde{\mathscr{D}}
$$

Remark 2.1. In the previous definitions, for each $l, l=1,2, \ldots, N_{B}$, the variables $z_{l}$ are assumed to be mutually independent.

On $\mathfrak{H}$ spanned by the bosonic eigenvectors $\left|\Phi_{\mathbf{n}}^{[\mathbf{k}]}\right\rangle$ and $\left|\Phi_{\mathbf{n}}^{\prime \mathbf{k}]}\right\rangle$, we have the following resolutions of the identity:

$$
\begin{aligned}
& \int_{\mathscr{D}}\left|\eta_{\mathbf{z}}\right\rangle\left\langle\eta_{\mathbf{z}}\right| d \mu(\mathbf{z}, \overline{\mathbf{z}}) \mathscr{N}\left(|\mathbf{z}|^{2}\right)=I_{\mathfrak{H}} \\
& \int_{\mathscr{D}}\left|\eta_{\mathbf{z}}^{\prime}\right\rangle\left\langle\eta_{\mathbf{z}}^{\prime}\right| d \mu(\mathbf{z}, \overline{\mathbf{z}}) \mathscr{N}^{\prime}\left(|\mathbf{z}|^{2}\right)=I_{\mathfrak{H}}
\end{aligned}
$$

The measure $d \mu(\mathbf{z}, \overline{\mathbf{z}})$ is such that $d \mu(\mathbf{z}, \overline{\mathbf{z}})=d \lambda(r) d \theta, \mathbf{z}=r e^{l \theta}$, where $d \lambda(r)$ is determined through the moment problem:

$$
2 \pi \int_{0}^{L} r^{2 \mathbf{n}} d \lambda(r)=\{\mathbf{n}\} !, \quad \mathbf{n}=0,1,2, \cdots
$$

in the case of the vectors $D_{\mathbf{n}}(\mathbf{z})|0\rangle$; for the vectors $D_{\mathbf{n}}^{\prime}(\mathbf{z})|0\rangle$, with $d \mu(\mathbf{z}, \overline{\mathbf{z}})=d \rho(r) d \theta$,

$$
2 \pi \int_{0}^{\tilde{L}} r^{2 \mathbf{n}} d \rho(r)=\{\mathbf{n}\}_{d} !, \quad \mathbf{n}=0,1,2, \cdots
$$




\section{Probability density computation}

This section discusses the probability density and its time evolution evaluated in the constructed NCS basis.

We start computing the quantity $\left\langle\eta_{\mathbf{z}} \mid \eta_{\mathbf{z}_{\mathbf{0}}}\right\rangle$. Making use of (2.57), (2.49) and (2.55)-(2.56), we obtain

$$
\begin{aligned}
\left\langle\eta_{\mathbf{z}} \mid \eta_{\mathbf{z}_{\mathbf{0}}}\right\rangle & =\left[\mathscr{N}\left(|\mathbf{z}|^{2}\right) \mathscr{N}\left(\left|\mathbf{z}_{\mathbf{0}}\right|^{2}\right)\right]^{-1 / 2} \sum_{\mathbf{n}=0}^{\infty} \frac{\left(\overline{\mathbf{z}} \mathbf{z}_{\mathbf{0}}\right)^{\mathbf{n}}}{\{\mathbf{n}\} !} \\
& =\frac{\mathscr{N}\left(\overline{\mathbf{z}} \mathbf{z}_{\mathbf{0}}\right)}{\sqrt{\mathscr{N}\left(|\mathbf{z}|^{2}\right) \mathscr{N}\left(\left|\mathbf{z}_{\mathbf{0}}\right|^{2}\right)}}
\end{aligned}
$$

giving

$$
\left|\left\langle\eta_{\mathbf{z}} \mid \eta_{\mathbf{z}_{\mathbf{0}}}\right\rangle\right|^{2}=\frac{\left|\mathscr{N}\left(\overline{\mathbf{z}} \mathbf{z}_{\mathbf{0}}\right)\right|^{2}}{\mathscr{N}\left(|\mathbf{z}|^{2}\right) \mathscr{N}\left(\left|\mathbf{z}_{\mathbf{0}}\right|^{2}\right)}, \quad\left|\mathscr{N}\left(\overline{\mathbf{z}} \mathbf{z}_{\mathbf{0}}\right)\right|^{2}:=\mathscr{N}\left(\overline{\mathbf{z}} \mathbf{z}_{\mathbf{0}}\right) \mathscr{N}\left(\overline{\mathbf{z}}_{0}\right)
$$

Then, the probability density is defined as the map

$$
\mathscr{D} \rightarrow \mathbb{R}_{+}, \quad \mathbf{z} \mapsto \rho_{\mathbf{z}_{0}}(\mathbf{z}):=\left|\left\langle\eta_{\mathbf{z}} \mid \eta_{\mathbf{z}_{0}}\right\rangle\right|^{2}=\frac{\left|\mathscr{N}\left(\overline{\mathbf{z}} \mathbf{z}_{\mathbf{0}}\right)\right|^{2}}{\mathscr{N}\left(|\mathbf{z}|^{2}\right) \mathscr{N}\left(\left|\mathbf{z}_{\mathbf{0}}\right|^{2}\right)}
$$

Consider the following spectral decomposition in the eigenstates $\left|\Phi_{\mathbf{n}}^{[\mathbf{k}]}\right\rangle$ given by

$$
\tilde{H}=\sum_{\mathbf{n}=0}^{\infty} \Omega_{N_{B}} \mathscr{E}_{\mathbf{n}}\left|\Phi_{\mathbf{n}}^{[\mathbf{k}]}\right\rangle\left\langle\Phi_{\mathbf{n}}^{[\mathbf{k}]}\right|, \quad \mathscr{E}_{\mathbf{n}}=\sum_{l=1}^{N_{B}} n_{l} f_{l}\left(n_{l}\right)
$$

with

$$
\Omega_{N_{B}}:=\left(\sum_{l=1}^{N_{B}} \omega_{l} n_{l} f_{l}\left(n_{l}\right)\right) /\left(\sum_{l=1}^{N_{B}} n_{l} f_{l}\left(n_{l}\right)\right) .
$$

Let

$$
\mathbf{J}^{\mathbf{n} / 2}:=\prod_{l=1}^{N_{B}} J_{l}^{n_{l} / 2}, \quad \varepsilon_{\mathbf{n}}:=\left(n_{1} f_{1}\left(n_{1}\right), n_{2} f_{2}\left(n_{2}\right), \cdots, n_{N_{B}} f_{N_{B}}\left(n_{N_{B}}\right)\right), \quad \gamma:=\left(\begin{array}{c}
\gamma_{1} \\
\gamma_{2} \\
\vdots \\
\gamma_{N_{B}}
\end{array}\right)
$$

where $\mathbf{z}_{\mathbf{0}}{ }^{\mathbf{n}}:=\mathbf{J}_{\mathbf{0}}{ }^{\mathbf{n} / 2} e^{-l \varepsilon_{\mathbf{n}} \gamma_{0}}$, such that we get 


$$
e^{-l \tilde{H} t}\left|\eta_{\mathbf{z}_{\mathbf{0}}}\right\rangle=\left(\mathscr{N}\left(\left|\mathbf{z}_{\mathbf{0}}\right|^{2}\right)\right)^{-1 / 2} \sum_{\mathbf{n}=0}^{\infty} \frac{\mathbf{J}_{\mathbf{0}} \mathbf{n} / 2}{e^{-l \varepsilon_{\mathbf{n}} \gamma_{0}}} e^{-l\left(\Omega_{N_{B}} \mathscr{E}_{\mathbf{n}}\right) t}\left|\Phi_{\mathbf{n}}^{[\mathbf{k}]}\right\rangle
$$

Since

$$
\varepsilon_{\mathbf{n}} \beta=\left(n_{1} f_{1}\left(n_{1}\right), n_{2} f_{2}\left(n_{2}\right), \cdots, n_{N_{B}} f_{N_{B}}\left(n_{N_{B}}\right)\right)\left(\begin{array}{c}
1 \\
\vdots \\
1 \\
\vdots \\
1
\end{array}\right)=\sum_{l=1}^{N_{B}} n_{l} f_{l}\left(n_{l}\right)=\mathscr{E}_{\mathbf{n}}
$$

we have

$$
e^{-l \tilde{H} t}\left|\eta_{\mathbf{z}_{\mathbf{0}}}\right\rangle=\left|\mathbf{J}_{0}, \gamma_{0}+\Omega_{N_{B}} t \beta\right\rangle=\left|\eta_{\mathbf{z}_{\mathbf{0}}(\mathbf{t})}\right\rangle, \quad\left(\mathbf{z}_{\mathbf{0}}(t)\right)^{\mathbf{n}}:=\mathbf{J}_{\mathbf{0}}^{\mathbf{n} / 2} e^{-l \varepsilon_{\mathbf{n}}\left(\gamma_{0}+\Omega_{N_{B}} t \beta\right)} .
$$

From (3.7) and (3.9), we obtain

$$
\begin{aligned}
\left\langle\eta_{\mathbf{z}}\left|e^{-i H t}\right| \eta_{\mathbf{z}_{\mathbf{0}}}\right\rangle= & {\left[\mathscr{N}\left(|\mathbf{z}|^{2}\right) \mathscr{N}\left(\left|\mathbf{z}_{\mathbf{0}}\right|^{2}\right)\right]^{-1 / 2} \sum_{\mathbf{m}, \mathbf{n}=0}^{\infty} \frac{\mathbf{J}^{\mathbf{m} / 2} e^{l \varepsilon_{[\mathbf{m}]} \gamma}}{\sqrt{\{\mathbf{m}\} !}} \frac{\mathbf{J}_{\mathbf{0}}^{\mathbf{n} / 2} e^{-l \varepsilon_{[\mathbf{n}]}\left(\gamma_{0}+\Omega_{N} t \beta\right)}}{\sqrt{\{\mathbf{n}\} !}} } \\
& \left\langle\Phi_{[\mathbf{m}]}^{[\mathbf{k}]} \mid \Phi_{[\mathbf{n}]}^{[\mathbf{k}]}\right\rangle \\
= & {\left[\mathscr{N}\left(|\mathbf{z}|^{2}\right) \mathscr{N}\left(\left|\mathbf{z}_{\mathbf{0}}\right|^{2}\right)\right]^{-1 / 2} \sum_{\mathbf{n}=0}^{\infty} \frac{\mathbf{J}^{\mathbf{n} / 2} \mathbf{J}_{\mathbf{0}}^{\mathbf{n} / 2} e^{-l \varepsilon_{[\mathbf{n}]}\left(\gamma_{0}+\Omega_{N} t \beta-\gamma\right)}}{\{\mathbf{n}\} !} }
\end{aligned}
$$

and thereby

$$
\left|\left\langle\eta_{\mathbf{z}}\left|e^{-i \tilde{H} t}\right| \eta_{\mathbf{z}_{\mathbf{0}}}\right\rangle\right|^{2}=\frac{\left|\mathscr{N}\left(\overline{\mathbf{z}} \mathbf{z}_{\mathbf{0}}(t)\right)\right|^{2}}{\mathscr{N}\left(|\mathbf{z}|^{2}\right) \mathscr{N}\left(\left|\mathbf{z}_{\mathbf{0}}\right|^{2}\right)}
$$

Thus, the time evolution behavior of $\rho_{z 0}(z)$ is provided by the mapping

$$
\mathbf{z} \mapsto \rho_{\mathbf{z}_{0}}(\mathbf{z}, t):=\left|\left\langle\eta_{\mathbf{z}}\left|e^{-i \tilde{H} t}\right| \eta_{\mathbf{z}_{0}}\right\rangle\right|^{2}=\frac{\left|\mathscr{N}\left(\overline{\mathbf{z}} \mathbf{z}_{\mathbf{0}}(t)\right)\right|^{2}}{\mathscr{N}\left(|\mathbf{z}|^{2}\right) \mathscr{N}\left(\left|\mathbf{z}_{\mathbf{0}}\right|^{2}\right)}
$$

while the dynamics of the NCS $\left|\eta_{\mathbf{z}}\right\rangle$ is governed by the relation:

$$
\left|\eta_{\mathbf{z}} ; t\right\rangle=e^{-i \tilde{H} t}\left|\eta_{\mathbf{z}}\right\rangle=\left|\eta_{\mathbf{z}(t)}\right\rangle, \quad(\mathbf{z}(t))^{\mathbf{n}}:=\mathbf{J}^{\mathbf{n} / 2} e^{-l \varepsilon_{\mathbf{n}}\left(\gamma+\Omega_{N_{B}} t \beta\right)}
$$

Finally, as

$$
\left\langle\eta_{\mathbf{z}}^{\prime} \mid \eta_{\mathbf{z}_{0}}^{\prime}\right\rangle=\left[\mathscr{N}\left(|\mathbf{z}|^{2}\right) \mathscr{N}\left(\left|\mathbf{z}_{\mathbf{0}}\right|^{2}\right)\right]^{-1 / 2} \sum_{\mathbf{n}=0}^{\infty} \frac{\left(\overline{\mathbf{z}} \mathbf{z}_{\mathbf{0}}\right)^{\mathbf{n}}}{\{\mathbf{n}\}_{d} !}
$$

the NCS $\left|\eta_{\mathbf{z}}^{\prime}\right\rangle$ obey the same relations as (3.12) and (3.13). 


\section{Reproducing kernel}

In this section, we discuss another important property of the NCS, i.e. the reproducing kernel on the Hilbert space $\mathfrak{H}$, based on the completeness relation (2.59).

From the expression (3.1), we introduce the quantity

$$
\mathscr{K}\left(\mathbf{z}, \mathbf{z}^{\prime}\right):=\left\langle\eta_{\mathbf{z}^{\prime}} \mid \eta_{\mathbf{z}}\right\rangle=\frac{\mathscr{N}\left(\overline{\left.\mathbf{z}^{\prime} \mathbf{z}\right)}\right.}{\sqrt{\mathscr{N}\left(\left|\mathbf{z}^{\prime}\right|^{2}\right) \mathscr{N}\left(|\mathbf{z}|^{2}\right)}}
$$

standing for a reproducing kernel [1]. Indeed,

\section{Proposition 4.1.}

The following properties are satisfied by the function $\mathscr{K}$ on the Hilbert space $\mathfrak{H}$ :

(i) Hermiticity

$$
\mathscr{K}\left(\mathbf{z}, \mathbf{z}^{\prime}\right)=\overline{\mathscr{K}\left(\mathbf{z}^{\prime}, \mathbf{z}\right)}
$$

(ii) Positivity

$$
\mathscr{K}(\mathbf{z}, \mathbf{z})>0
$$

(iii) Idempotence

$$
\int_{\mathscr{D}} d \mu\left(\mathbf{z}^{\prime \prime}, \overline{\mathbf{z}^{\prime \prime}}\right) \mathscr{K}\left(\mathbf{z}, \mathbf{z}^{\prime \prime}\right) \mathscr{K}\left(\mathbf{z}^{\prime \prime}, \mathbf{z}^{\prime}\right)=\mathscr{K}\left(\mathbf{z}, \mathbf{z}^{\prime}\right) .
$$

The condition (4.4), also called the square integrability property of the reproducing kernel [1], is a consequence of (2.59).

Proof. See Appendix.

For any $|\Psi\rangle \in \mathfrak{H}$, we have

$$
|\Psi\rangle=\int_{\mathscr{D}} d \mu(\mathbf{z}, \overline{\mathbf{z}}) \Psi(\mathbf{z})\left|\eta_{\mathbf{z}}\right\rangle
$$

where $\Psi(\mathbf{z}):=\left\langle\eta_{\mathbf{z}} \mid \Psi\right\rangle$. The following reproducing property

$$
\Psi(\mathbf{z})=\int_{\mathscr{D}} d \mu\left(\mathbf{z}^{\prime}, \overline{\mathbf{z}^{\prime}}\right) \mathscr{K}\left(\mathbf{z}, \mathbf{z}^{\prime}\right) \Psi\left(\mathbf{z}^{\prime}\right)
$$

is immediate from the definition of the reproducing kernel (4.1).

\section{Diagonal representation of the density matrix}

In quantum mechanics, the probability distribution on the states of a physical system can be characterized by a statistical operator called density matrix, generally denoted by $\rho$. The latter reveals to be an important tool used for examining the physical and chemical properties of a system (see for e.g. [5] and references listed therein). In the Fock representation, the density matrix is given by its matrix elements, $\rho_{m, n}=\langle m|\rho| n\rangle$, as [13]: $\rho=\sum_{m, n} \rho_{m, n}|m\rangle\langle n|$. In this section, we investigate 
the statistical properties of the NCS making use of the density matrix. The results issued from the previous section are exploited as key ingredients to construct a Glauber-Sudarshan $P$-representation of the density matrix following $[9,10,13]$ and [24], where a $q$-analogue has been performed. We first provide this $P$-representation in the electron-phonon system bosonic states and NCS basis, and then extend it to matrix domain with respect to the physical model VCS basis.

Assuming that our quantum model obeys the conditions of the quantum canonical distribution, the density matrix, for a fixed $[\mathbf{k}]$, is given, in terms of the bosonic states, as

$$
\rho_{[\mathbf{k}]}=\sum_{\mathbf{n}, \mathbf{m}=0}^{\infty} \rho_{[\mathbf{k}]}(\mathbf{n}, \mathbf{m})\left|\Phi_{\mathbf{n}}^{[\mathbf{k}]}\right\rangle\left\langle\Phi_{\mathbf{m}}^{[\mathbf{k}]}\right|
$$

where the $\rho_{[\mathbf{k}]}(\mathbf{n}, \mathbf{m})$ are the matrix elements.

In a matrix form, we get, by using (5.1), the following expression for $\hat{\rho}$ :

$$
\hat{\rho}=\sum_{[\mathbf{k}] \in \Gamma \mathbf{n}, \mathbf{m}=0} \sum_{[\mathbf{k}]}^{\infty}(\mathbf{n}, \mathbf{m})\left|\Psi_{[\mathbf{k}]} \otimes \Phi_{[\mathbf{n}]}^{[\mathbf{k}]}\right\rangle\left\langle\Psi_{[\mathbf{k}]} \otimes \Phi_{[\mathbf{m}]}^{[\mathbf{k}]}\right|
$$

which takes on the Hilbert space $\mathbb{C}^{2^{M}} \otimes \mathfrak{H}$ the form

$$
\hat{\rho}=\operatorname{diag}\left(\rho_{(0,0, \ldots, 0,0)}, \rho_{(1,0, \ldots 0,0)}, \cdots, \rho_{[\mathbf{k}]}, \cdots, \rho_{(1,1, \ldots, 1,1)}\right)
$$

where $[\mathbf{n}]:=n_{1}, n_{2}, \ldots, n_{N_{B}}$ and $\mathfrak{H}=\bigoplus_{[\mathbf{k}] \in \Gamma} \mathfrak{H}_{[\mathbf{k}]}, \mathfrak{H}_{[\mathbf{k}]}$ is the subspace of $\mathfrak{H}$ spanned by the vectors $\left|\Phi_{[\mathbf{n}]}^{[\mathbf{k}]}\right\rangle$ with $\sum_{[\mathbf{k}] \in \Gamma}\left|\Psi_{[\mathbf{k}]}\right\rangle\left\langle\Psi_{[\mathbf{k}]}\right|=\mathbb{I}_{2^{M}}$ with $\Gamma$ defined in (2.7).

Keeping in mind the radial parametrization $\mathbf{z}=r e^{\imath \theta}$ we get the following quantity $\mathscr{N}\left(|\mathbf{z}|^{2}\right)\left|r e^{\imath \theta}\right\rangle\left\langle r e^{\imath \theta}\right|:$

$$
\mathscr{N}\left(|\mathbf{z}|^{2}\right)\left|r e^{\imath \theta}\right\rangle\left\langle r e^{\imath \theta}\left|=\sum_{\mathbf{n}, \mathbf{m}=0}^{\infty} \frac{r^{\mathbf{m}+\mathbf{n}} e^{l(\mathbf{n}-\mathbf{m}) \theta}}{\sqrt{\{\mathbf{n}\} !\{\mathbf{m}\} !}}\right| \Phi_{\mathbf{n}}^{[\mathbf{k}]}\right\rangle\left\langle\Phi_{\mathbf{m}}^{[\mathbf{k}]}\right|
$$

which leads to the integral

$$
\int_{0}^{2 \pi} \frac{d \theta}{2 \pi} e^{\imath \mathbf{l} \theta} \mathscr{N}\left(|\mathbf{z}|^{2}\right)\left|r e^{\imath \theta}\right\rangle\left\langle r e^{\imath \theta}\left|=\sum_{\mathbf{n}, \mathbf{m}=0}^{\infty} \frac{r^{\mathbf{m}+\mathbf{n}}}{\sqrt{\{\mathbf{n}\} !\{\mathbf{m}\} !}} \delta(\mathbf{n}-\mathbf{m}+\mathbf{l})\right| \Phi_{\mathbf{n}}^{[\mathbf{k}]}\right\rangle\left\langle\Phi_{\mathbf{m}}^{[\mathbf{k}]}\right| .
$$

We obtain the expression

$$
\begin{aligned}
\left(\frac{d}{d r}\right)^{\mathbf{q}} \int_{0}^{2 \pi} \frac{d \theta}{2 \pi} e^{l l \theta} \mathscr{N}\left(|\mathbf{z}|^{2}\right)\left|r e^{\imath \theta}\right\rangle\left\langle r e^{\iota \theta}\right|= & \sum_{\mathbf{n}, \mathbf{m}=0}^{\infty} \frac{r^{\mathbf{m}+\mathbf{n}-\mathbf{q}}}{\sqrt{\{\mathbf{n}\} !\{\mathbf{m}\} !}} \frac{(\mathbf{m}+\mathbf{n}) !}{(\mathbf{m}+\mathbf{n}-\mathbf{q}) !} \\
& \times \delta(\mathbf{n}-\mathbf{m}+\mathbf{l})\left|\Phi_{\mathbf{n}}^{[\mathbf{k}]}\right\rangle\left\langle\Phi_{\mathbf{m}}^{[\mathbf{k}]}\right|
\end{aligned}
$$

such that the following quantity

$$
\left\{\left(\frac{d}{d r}\right)^{\mathbf{q}} \int_{0}^{2 \pi} \frac{d \theta}{2 \pi} e^{l \mathbf{l} \theta} \mathscr{N}\left(|\mathbf{z}|^{2}\right)\left|r e^{\imath \theta}\right\rangle\left\langle r e^{\imath \theta}\right|\right\}_{r=0},
$$

where, at $r=0$ in the right-hand side of (5.6), the term with $\mathbf{m}+\mathbf{n}-\mathbf{q}=0$ alone survives, provides 


$$
\left|\Phi_{\mathbf{n}}^{[\mathbf{k}]}\right\rangle\left\langle\Phi_{\mathbf{m}}^{[\mathbf{k}]}\left|=\frac{\sqrt{\{\mathbf{n}\} !\{\mathbf{m}\} !}}{(\mathbf{m}+\mathbf{n}) !}\left(\frac{d}{d r}\right)^{\mathbf{m}+\mathbf{n}} \int_{0}^{2 \pi} \frac{d \theta}{2 \pi} e^{l(\mathbf{m}-\mathbf{n}) \theta} \mathscr{N}\left(|\mathbf{z}|^{2}\right)\right| r e^{\imath \theta}\right\rangle\left\langle r e^{\imath \theta}||_{r=0}\right.
$$

Then, a $f$-deformed Glauber-Sudarshan $P$-representation of the density matrix in the bosonic states basis is given by

$$
\rho_{[\mathbf{k}]}=\sum_{\mathbf{n}, \mathbf{m}=0}^{\infty} \rho_{[\mathbf{k}]}(\mathbf{n}, \mathbf{m}) \frac{\sqrt{\{\mathbf{n}\} !\{\mathbf{m}\} !}}{(\mathbf{m}+\mathbf{n}) !}\left(\frac{d}{d r}\right)^{\mathbf{m}+\mathbf{n}} \int_{0}^{2 \pi} \frac{d \theta}{2 \pi} e^{l(\mathbf{m}-\mathbf{n}) \theta} \mathscr{N}\left(|\mathbf{z}|^{2}\right)\left|r e^{\imath \theta}\right\rangle\left\langle r e^{l \theta}||_{r=0}\right.
$$

In the case of the dual NCS $\left|\eta_{\mathbf{z}}^{\prime}\right\rangle$, we get

$$
\left|\Phi_{\mathbf{n}}^{[\mathbf{k}]}\right\rangle\left\langle\Phi_{\mathbf{m}}^{[\mathbf{k}]}\left|=\frac{\sqrt{\{\mathbf{n}\}_{d} !\{\mathbf{m}\}_{d} !}}{(\mathbf{m}+\mathbf{n}) !}\left(\frac{d}{d r}\right)^{\mathbf{m}+\mathbf{n}} \int_{0}^{2 \pi} \frac{d \theta}{2 \pi} e^{l(\mathbf{m}-\mathbf{n}) \theta} \mathscr{N}\left(|\mathbf{z}|^{2}\right)\right| r e^{\imath \theta}\right\rangle\left\langle r e^{l \theta}||_{r=0},\right.
$$

and

$$
\tilde{\rho}_{[\mathbf{k}]}=\sum_{\mathbf{n}, \mathbf{m}=0}^{\infty} \tilde{\rho}_{[\mathbf{k}]}(\mathbf{n}, \mathbf{m}) \frac{\sqrt{\{\mathbf{n}\}_{d} !\{\mathbf{m}\}_{d} !}}{(\mathbf{m}+\mathbf{n}) !}\left(\frac{d}{d r}\right)^{\mathbf{m}+\mathbf{n}} \int_{0}^{2 \pi} \frac{d \theta}{2 \pi} e^{l(\mathbf{m}-\mathbf{n}) \theta} \mathscr{N}\left(|\mathbf{z}|^{2}\right)\left|r e^{\imath \theta}\right\rangle\left\langle r e^{\imath \theta}||_{r=0} .\right.
$$

Using the definition (5.1), the matrix elements of $\rho_{[\mathbf{k}]}$ computed with two NCS is found to be

$$
\left\langle\eta_{\mathbf{z}^{\prime}}\left|\rho_{[\mathbf{k}]}\right| \eta_{\mathbf{z}}\right\rangle=\left[\mathscr{N}\left(\left|\mathbf{z}^{\prime}\right|^{2}\right) \mathscr{N}\left(|\mathbf{z}|^{2}\right)\right]^{-1 / 2} \sum_{\mathbf{m}, \mathbf{n}=0}^{\infty} \rho_{[\mathbf{k}]}(\mathbf{n}, \mathbf{m}) \frac{\overline{\mathbf{z}}^{\prime \mathbf{n}}}{\sqrt{\{\mathbf{n}\} !}} \frac{\mathbf{z}^{\mathbf{m}}}{\sqrt{\{\mathbf{m}\} !}}
$$

which corresponds at $\mathbf{z}^{\prime}=\mathbf{z}$, to the diagonal element

$$
\left\langle\eta_{\mathbf{z}}\left|\rho_{[\mathbf{k}]}\right| \eta_{\mathbf{z}}\right\rangle=\left(\mathscr{N}\left(|\mathbf{z}|^{2}\right)\right)^{-1} \sum_{\mathbf{m}, \mathbf{n}=0}^{\infty} \rho_{[\mathbf{k}]}(\mathbf{n}, \mathbf{m}) \frac{\overline{\mathbf{z}}^{\mathbf{n}}}{\sqrt{\{\mathbf{n}\} !}} \frac{\mathbf{z}^{\mathbf{m}}}{\sqrt{\{\mathbf{m}\} !}} .
$$

The quantity $\left\langle\eta_{\mathbf{z}}\left|\rho_{[\mathbf{k}]}\right| \eta_{\mathbf{z}}\right\rangle$ is analog to the "semi-classical "phase space distribution function $\mu(x, p)=\langle z|\rho| z\rangle$ associated to the density matrix $\rho$ (here $\rho_{[\mathbf{k}]}$ ) of the system which is normalized as $\int(d x d p / 2 \pi \hbar) \mu(x, p)=1$, and often referred to as the Husimi distribution [15]. Remark that an immediate issue of the representations (5.12) and (5.13) with respect to the results of Section 4, will be evidenced in the use of (5.12) when discussing the reproducing kernel insights of the density matrix.

In terms of the NCS, the density matrix is given by

$$
\rho_{[\mathbf{k}]}=\int_{\mathscr{D}} d \mu(\mathbf{z}, \overline{\mathbf{z}}) \mathscr{N}\left(|\mathbf{z}|^{2}\right) P\left(|\mathbf{z}|^{2}\right)\left|\eta_{\mathbf{z}}\right\rangle\left\langle\eta_{\mathbf{z}}\right|,
$$

where $P\left(|\mathbf{z}|^{2}\right)$ satisfies the normalization condition 


$$
\int_{\mathscr{D}} d \mu(\mathbf{z}, \overline{\mathbf{z}}) P\left(|\mathbf{z}|^{2}\right)=1
$$

Note that (5.14) is exploited to derive the quantum-statistical or thermal averages for an operator as shown in [5] (see also references therein).

Then,

$$
\left\langle\Phi_{\mathbf{n}}^{[\mathbf{k}]}\left|\rho_{[\mathbf{k}]}\right| \Phi_{\mathbf{n}}^{[\mathbf{k}]}\right\rangle=\int_{\mathscr{D}} d \mu(\mathbf{z}, \overline{\mathbf{z}}) \mathscr{N}\left(|\mathbf{z}|^{2}\right) P\left(|\mathbf{z}|^{2}\right)\left|\left\langle\Phi_{\mathbf{n}}^{[\mathbf{k}]} \mid \eta_{\mathbf{z}}\right\rangle\right|^{2}
$$

where the quantity $\left|\left\langle\Phi_{\mathbf{n}}^{[\mathbf{k}]} \mid \eta_{\mathbf{z}}\right\rangle\right|^{2}$ calculated by use of (2.57) as follows:

$$
\begin{aligned}
\left|\left\langle\Phi_{\mathbf{n}}^{[\mathbf{k}]} \mid \eta_{\mathbf{z}}\right\rangle\right|^{2}= & \left\langle\Phi_{\mathbf{n}}^{[\mathbf{k}]} \mid \eta_{\mathbf{z}}\right\rangle\left\langle\eta_{\mathbf{z}} \mid \Phi_{\mathbf{n}}^{[\mathbf{k}]}\right\rangle \\
= & \left\{\left\langle\Phi_{\mathbf{n}}^{[\mathbf{k}]}\left|\left(\mathscr{N}\left(|\mathbf{z}|^{2}\right)\right)^{-1 / 2} \sum_{\mathbf{j}=0}^{\infty} \frac{\mathbf{z}^{\mathbf{j}}}{\sqrt{\{\mathbf{j}\} !}}\right| \Phi_{\mathbf{j}}^{[\mathbf{k}]}\right\rangle\right\} \\
& \times\left\{\left(\mathscr{N}\left(|\mathbf{z}|^{2}\right)\right)^{-1 / 2} \sum_{\mathbf{p}=0}^{\infty}\left\langle\Phi_{\mathbf{p}}^{[\mathbf{k}]}\left|\frac{\overline{\mathbf{z}}^{\mathbf{p}}}{\sqrt{\{\mathbf{p}\} !}}\right| \Phi_{\mathbf{n}}^{[\mathbf{k}]}\right\rangle\right\} \\
= & \left\{\left(\mathscr{N}\left(|\mathbf{z}|^{2}\right)\right)^{-1 / 2} \frac{\mathbf{z}^{\mathbf{n}}}{\sqrt{\{\mathbf{n}\} !}}\right\} \times\left\{\left(\mathscr{N}\left(|\mathbf{z}|^{2}\right)\right)^{-1 / 2} \frac{\overline{\mathbf{z}}^{\mathbf{n}}}{\sqrt{\{\mathbf{n}\} !}}\right\} \\
= & \left(\mathscr{N}\left(|\mathbf{z}|^{2}\right)\right)^{-1} \frac{|\mathbf{z}|^{2 \mathbf{n}}}{\{\mathbf{n}\} !}
\end{aligned}
$$

corresponds to a $f$-deformed photon number equal to

$$
\mathscr{P}(\mathbf{n}, \mathbf{z})=e^{-|\mathbf{z}|^{2}} \frac{|\mathbf{z}|^{2 \mathbf{n}}}{\mathbf{n} !},
$$

when $f(\mathbf{n}) \rightarrow 1$, which is a Poisson distribution. Therefore, we say that the NCS obey a $f$-Poisson distribution.

In the VCS formalism, we write that

$$
\hat{\rho}=\sum_{[\mathbf{k}] \in \Gamma} \int_{\mathscr{D}^{2 M}} d \mu(\mathfrak{Z}, \overline{\mathfrak{Z}}) \mathscr{N}\left(\left|\mathbf{z}_{[\mathbf{k}]}\right|^{2}\right) P\left(\left|\mathbf{z}_{[\mathbf{k}]}\right|^{2}\right)\left|\eta_{\mathfrak{Z}} ;[\mathbf{k}]\right\rangle\left\langle\eta_{\mathfrak{Z}} ;[\mathbf{k}]\right|
$$

where

$$
\left|\eta_{3} ;[\mathbf{k}]\right\rangle=\left(\begin{array}{c}
0 \\
\vdots \\
0 \\
\left|\eta_{\left.\mathbf{z}_{[\mathbf{k}]}\right]}\right\rangle \\
\vdots \\
0
\end{array}\right)
$$

coincides, in the special case of $f\left(N_{i}\right)=1\left(1 \leq i \leq N_{B}\right)$, with the VCS developed in [4], and 


$$
\left|\eta_{\mathbf{z}_{[\mathbf{k}]}}\right\rangle=:\left(\mathscr{N}\left(\left|\mathbf{z}_{[\mathbf{k}]}\right|^{2}\right)\right)^{-1 / 2} \sum_{\mathbf{n}=0}^{\infty} \frac{\mathbf{z}_{[\mathbf{k}]}^{\mathbf{n}}}{\sqrt{\{\mathbf{n}\} !}}\left|\Psi_{[\mathbf{k}]} \otimes \Phi_{[\mathbf{n}]}^{[\mathbf{k}]}\right\rangle
$$

such that, with respect to the measure $d \mu(\mathfrak{Z}, \overline{\mathfrak{Z}})=\prod_{[\mathbf{k}] \in \Gamma} d \mu\left(\mathbf{z}_{[\mathbf{k}]}, \overline{\mathbf{z}}_{[\mathbf{k}]}\right)$, the resolution of the identity provided as in [4],

$$
\begin{aligned}
\sum_{[\mathbf{k}] \in \Gamma} \int_{\mathscr{D}^{2}} d \mu(\mathfrak{Z}, \overline{\mathfrak{Z}}) \mathscr{N}\left(\left|\mathbf{z}_{[\mathbf{k}]}\right|^{2}\right)\left|\eta_{\mathfrak{Z}} ;[\mathbf{k}]\right\rangle\left\langle\eta_{\mathfrak{Z}} ;[\mathbf{k}]\right| & \left.=\sum_{[\mathbf{k}] \in \Gamma[\mathbf{n}]=0} \sum_{[\mathbf{k}]}^{\infty} \otimes \Phi_{[\mathbf{n}]}^{[\mathbf{k}]}\right\rangle\left\langle\Psi_{[\mathbf{k}]} \otimes \Phi_{[\mathbf{n}]}^{[\mathbf{k}]}\right| \\
& =\mathbb{I}_{2^{M}} \otimes I_{\mathfrak{H}},
\end{aligned}
$$

is satisfied.

Keeping in mind the following expression

$$
\left|\eta_{\mathbf{z}}\right\rangle\left\langle\eta_{\mathbf{z}}\left|=\left(\mathscr{N}\left(|\mathbf{z}|^{2}\right)\right)^{-1} \sum_{\mathbf{j}, \mathbf{p}=0}^{\infty} \frac{\mathbf{z}^{\mathbf{j}}}{\sqrt{\{\mathbf{j}\} !}} \frac{\overline{\mathbf{z}}^{\mathbf{p}}}{\sqrt{\{\mathbf{p}\} !}}\right| \Phi_{\mathbf{j}}^{[\mathbf{k}]}\right\rangle\left\langle\Phi_{\mathbf{p}}^{[\mathbf{k}]}\right|
$$

and using the relation (5.14), the density matrix can be explicitly computed as

$$
\rho_{[\mathbf{k}]}=\sum_{\mathbf{j}, \mathbf{p}=0}^{\infty} \int_{\mathscr{D}} d \mu(\mathbf{z}, \overline{\mathbf{z}}) P\left(|\mathbf{z}|^{2}\right)\left\{\frac{\mathbf{z}^{\mathbf{j}}}{\sqrt{\{\mathbf{j}\} !}} \frac{\overline{\mathbf{z}}^{\mathbf{p}}}{\sqrt{\{\mathbf{p}\} !}}\left|\Phi_{\mathbf{j}}^{[\mathbf{k}]}\right\rangle\left\langle\Phi_{\mathbf{p}}^{[\mathbf{k}]}\right|\right\} .
$$

From (5.1) and (5.24) together, it follows that

$$
\left\langle\Phi_{\mathbf{n}}^{[\mathbf{k}]}\left|\rho_{[\mathbf{k}]}\right| \Phi_{\mathbf{n}}^{[\mathbf{k}]}\right\rangle:=\rho_{[\mathbf{k}]}(\mathbf{n}, \mathbf{n})=\int_{\mathscr{D}} d \mu(\mathbf{z}, \overline{\mathbf{z}}) P\left(|\mathbf{z}|^{2}\right) \frac{|\mathbf{z}|^{2 \mathbf{n}}}{\{\mathbf{n}\} !},
$$

and the matrix elements of the density operator evaluated in the bosonic states $\left|\Phi_{\mathbf{n}}^{[\mathbf{k}]}\right\rangle$ and $\left|\Phi_{\mathbf{m}}^{[\mathbf{k}]}\right\rangle$ are provided by the expression

$$
\left\langle\Phi_{\mathbf{n}}^{[\mathbf{k}]}\left|\rho_{[\mathbf{k}]}\right| \Phi_{\mathbf{m}}^{[\mathbf{k}]}\right\rangle:=\rho_{[\mathbf{k}]}(\mathbf{n}, \mathbf{m})=\int_{\mathscr{D}} d \mu(\mathbf{z}, \overline{\mathbf{z}}) P\left(|\mathbf{z}|^{2}\right)\left\{\frac{\mathbf{z}^{\mathbf{n}}}{\sqrt{\{\mathbf{n}\} !}} \frac{\overline{\mathbf{z}}^{\mathbf{m}}}{\sqrt{\{\mathbf{m}\} !}}\right\}
$$

which reduces to (5.25) at $\mathbf{m}=\mathbf{n}$.

Taking

$$
\rho_{[\mathbf{k}]}=\int_{\mathscr{D}} d \mu(\mathbf{z}, \overline{\mathbf{z}}) P\left(|\mathbf{z}|^{2}\right)\left|\eta_{\mathbf{z}}\right\rangle\left\langle\eta_{\mathbf{z}}\right|
$$

instead of the relation (5.14), giving explicitly

$$
\rho_{[\mathbf{k}]}=\int_{\mathscr{D}} d \mu(\mathbf{z}, \overline{\mathbf{z}}) P\left(|\mathbf{z}|^{2}\right)\left|\eta_{\mathbf{z}}\right\rangle\left\langle\eta_{\mathbf{z}}\right|
$$




$$
\begin{aligned}
& =\int_{0}^{L} \int_{0}^{2 \pi} d \lambda(r) d \theta P\left(|\mathbf{z}|^{2}\right)\left\{\left(\mathscr{N}\left(|\mathbf{z}|^{2}\right)\right)^{-1} \sum_{\mathbf{j}, \mathbf{p}=0}^{\infty} \frac{\mathbf{z}^{\mathbf{j}}}{\sqrt{\{\mathbf{j}\} !}} \frac{\overline{\mathbf{z}}^{\mathbf{p}}}{\sqrt{\{\mathbf{p}\} !}}\left|\Phi_{\mathbf{j}}^{[\mathbf{k}]}\right\rangle\left\langle\Phi_{\mathbf{p}}^{[\mathbf{k}]}\right|\right\} \\
& =\sum_{\mathbf{j}, \mathbf{p}=0}^{\infty} 2 \pi \int_{0}^{L} r^{(\mathbf{j}+\mathbf{p})} d \lambda(r) P\left(r^{2}\right)\left(\mathscr{N}\left(r^{2}\right)\right)^{-1} \frac{\left|\Phi_{\mathbf{j}}^{[\mathbf{k}]}\right\rangle\left\langle\Phi_{\mathbf{p}}^{[\mathbf{k}]}\right|}{\sqrt{\{\mathbf{j}\} !\{\mathbf{p}\} !}} \delta_{\mathbf{j p}}
\end{aligned}
$$

and using (5.1) and (5.24) providing

$$
\begin{aligned}
\left\langle\Phi_{\mathbf{n}}^{[\mathbf{k}]}\left|\rho_{[\mathbf{k}]}\right| \Phi_{\mathbf{m}}^{[\mathbf{k}]}\right\rangle & :=\rho_{[\mathbf{k}]}(\mathbf{n}, \mathbf{m}) \\
& =\left\langle\Phi_{\mathbf{n}}^{[\mathbf{k}]}\left|\left\{\sum_{\mathbf{j}, \mathbf{p}=0}^{\infty} 2 \pi \int_{0}^{L} r^{(\mathbf{j}+\mathbf{p})} d \lambda(r) P\left(r^{2}\right)\left(\mathscr{N}\left(r^{2}\right)\right)^{-1} \frac{\left|\Phi_{\mathbf{j}}^{[\mathbf{k}]}\right\rangle\left\langle\Phi_{\mathbf{p}}^{[\mathbf{k}]}\right|}{\sqrt{\{\mathbf{j}\} !\{\mathbf{p}\} !}} \delta_{\mathbf{j p}}\right\}\right| \Phi_{\mathbf{m}}^{[\mathbf{k}]}\right\rangle \\
& =2 \pi \int_{0}^{L} r^{(\mathbf{n}+\mathbf{m})} d \lambda(r) P\left(r^{2}\right)\left(\mathscr{N}\left(r^{2}\right)\right)^{-1}\left[\frac{\delta_{\mathbf{n m}}}{\sqrt{\{\mathbf{n}\} !\{\mathbf{m}\} !}}\right]
\end{aligned}
$$

the expression (5.25) can be rewritten as

$$
\left\langle\Phi_{\mathbf{n}}^{[\mathbf{k}]}\left|\rho_{[\mathbf{k}]}\right| \Phi_{\mathbf{n}}^{[\mathbf{k}]}\right\rangle:=\rho_{[\mathbf{k}]}(\mathbf{n}, \mathbf{n})=\int_{\mathscr{D}} d \mu(\mathbf{z}, \overline{\mathbf{z}}) P\left(|\mathbf{z}|^{2}\right)\left(\mathscr{N}\left(|\mathbf{z}|^{2}\right)\right)^{-1} \frac{|\mathbf{z}|^{2 \mathbf{n}}}{\{\mathbf{n}\} !} .
$$

Therefore, by using (5.15), we obtain

$$
\sum_{\mathbf{n}=0}^{\infty}\left\langle\Phi_{\mathbf{n}}^{[\mathbf{k}]}\left|\rho_{[\mathbf{k}]}\right| \Phi_{\mathbf{n}}^{[\mathbf{k}]}\right\rangle=\int_{\mathscr{D}} d \mu(\mathbf{z}, \overline{\mathbf{z}}) P\left(|\mathbf{z}|^{2}\right)\left\{\left(\mathscr{N}\left(|\mathbf{z}|^{2}\right)\right)^{-1} \sum_{\mathbf{n}=0}^{\infty} \frac{|\mathbf{z}|^{2 \mathbf{n}}}{\{\mathbf{n}\} !}\right\}=1
$$

which is in accordance with the density matrix trace condition $\operatorname{Tr} \rho_{[\mathbf{k}]}:=\sum_{\mathbf{n}=0}^{\infty}\left\langle\Phi_{\mathbf{n}}^{[\mathbf{k}]}\left|\rho_{[\mathbf{k}]}\right| \Phi_{\mathbf{n}}^{[\mathbf{k}]}\right\rangle=$ 1.

From the resolution of the identity and (5.12), we derive the reproducing kernel property of the density matrix (see [24]) by setting $\left\langle\eta_{\mathbf{z}^{\prime}}\left|\rho_{[\mathbf{k}]}\right| \eta_{\mathbf{z}}\right\rangle:=\rho_{[\mathbf{k}]}\left(\mathbf{z}^{\prime}, \mathbf{z}\right)$, i.e.

$$
\begin{aligned}
\rho_{[\mathbf{k}]}\left(\mathbf{z}^{\prime}, \mathbf{z}\right) & =\left\langle\eta_{\mathbf{z}^{\prime}}\left|\int_{\mathscr{D}} d \mu\left(\mathbf{z}^{\prime \prime}, \overline{\mathbf{z}^{\prime \prime}}\right)\right| \eta_{\mathbf{z}^{\prime \prime}}\right\rangle\left\langle\eta_{\mathbf{z}^{\prime \prime}}\left|\rho_{[\mathbf{k}]}\right| \eta_{\mathbf{z}}\right\rangle \\
& =\int_{\mathscr{D}} d \mu\left(\mathbf{z}^{\prime \prime}, \overline{\mathbf{z}^{\prime \prime}}\right) \mathscr{K}\left(\mathbf{z}^{\prime \prime}, \mathbf{z}^{\prime}\right) \rho_{[\mathbf{k}]}\left(\mathbf{z}^{\prime \prime}, \mathbf{z}\right) .
\end{aligned}
$$

By making use of the Proposition 4.1 and relation (5.32), we infer here that the density matrix also displays the self-reproducing kernel properties as provided below:

\section{Proposition 5.1.}

The following properties are satisfied by $\rho_{[\mathbf{k}]}$ on the Hilbert space $\mathfrak{H}$ :

(i) Hermiticity

$$
\rho_{[\mathbf{k}]}\left(\mathbf{z}, \mathbf{z}^{\prime}\right)=\overline{\rho_{[\mathbf{k}]}\left(\mathbf{z}^{\prime}, \mathbf{z}\right)}
$$

(ii) Positivity

$$
\rho_{[\mathbf{k}]}(\mathbf{z}, \mathbf{z})>0
$$


(iii) Idempotence

$$
\int_{\mathscr{D}} d \mu\left(\mathbf{z}^{\prime \prime}, \overline{\mathbf{z}^{\prime \prime}}\right) \rho_{[\mathbf{k}]}\left(\mathbf{z}, \mathbf{z}^{\prime \prime}\right) \rho_{[\mathbf{k}]}\left(\mathbf{z}^{\prime \prime}, \mathbf{z}^{\prime}\right)=\rho_{[\mathbf{k}]}\left(\mathbf{z}, \mathbf{z}^{\prime}\right)
$$

Proof. See Appendix.

Coming back to (5.19), setting $\mathbf{z}^{\mathbf{n}}:=\mathbf{J}^{\mathbf{n} / 2} e^{-l \varepsilon_{[\mathbf{n}]} \gamma}$, and with

$$
\left|\eta_{\left.\mathbf{z}_{[\mathbf{k}]}\right\rangle}\right\rangle\left\langle\eta_{\left.\mathbf{z}_{[\mathbf{k}]}\right]}\left|=\left(\mathscr{N}\left(\left|\mathbf{z}_{[\mathbf{k}]}\right|^{2}\right)\right)^{-1} \sum_{[\mathbf{m}],[\mathbf{n}]=0}^{\infty} \frac{\mathbf{z}_{[\mathbf{k}]}^{\mathbf{n}}}{\sqrt{\{\mathbf{n}\} !}} \frac{\overline{\mathbf{z}}_{[\mathbf{k}]}^{\mathbf{m}}}{\sqrt{\{\mathbf{m}\} !}}\right| \Psi_{[\mathbf{k}]} \otimes \Phi_{[\mathbf{n}]}^{[\mathbf{k}]}\right\rangle\left\langle\Psi_{[\mathbf{k}]} \otimes \Phi_{[\mathbf{m}]}^{[\mathbf{k}]}\right|
$$

$d \mu(\mathfrak{Z}, \overline{\mathfrak{Z}})=d \mu(\gamma) d v(\mathfrak{J})$ where $d \mu(\gamma)=\prod_{[\mathbf{k}] \in \Gamma} d \mu\left(\gamma_{[\mathbf{k}]}\right), d v(\mathfrak{J})=\prod_{[\mathbf{k}] \in \Gamma} d v\left(\mathbf{J}_{[\mathbf{k}]}\right)$, and taking $\mathscr{D}=([0, \infty) \times[0,2 \pi))^{N_{B}}$, we get

$$
\begin{aligned}
& \hat{\rho}=\sum_{[\mathbf{k}] \in \Gamma[\mathbf{j}],[\mathbf{q}]=0} \sum_{\mathscr{D}}^{\infty} d \mu\left(\gamma_{(0, \cdots, 0,0)}\right) d v_{(0, \cdots, 0,0)}\left(\mathbf{J}_{(0, \cdots, 0,0)}\right) \int_{\mathscr{D}} d \mu\left(\gamma_{(1, \cdots, 0,0)}\right) d v_{(1, \cdots, 0,0)}\left(\mathbf{J}_{(1, \cdots, 0,0)}\right) \cdots \\
& \int_{\mathscr{D}} d \mu\left(\gamma_{[\mathbf{k}]}\right) d v_{[\mathbf{k}]}\left(\mathbf{J}_{[\mathbf{k}]}\right) \cdots \int_{\mathscr{D}} d \mu\left(\gamma_{(1, \cdots, 1,1)}\right) d v_{(1, \cdots, 1,1)}\left(\mathbf{J}_{(1, \cdots, 1,1)}\right) \times \\
& \operatorname{diag}\left(\frac{\mathbf{J}_{(0,0, \ldots, 0,0)}^{(\mathbf{j}+\mathbf{q}) / 2} e^{-l\left(\varepsilon_{[\mathbf{j}]}-\varepsilon_{[\mathbf{q}]}\right) \gamma_{(0,0, \ldots, 0,0)}} P\left(\mathbf{J}_{(0,0, \ldots, 0,0)}\right)}{\sqrt{\{\mathbf{j}\} !\{\mathbf{q}\} !}}, \frac{\mathbf{J}_{(1,0, \ldots 0,0)}^{(\mathbf{j}+\mathbf{q}) / 2} e^{-l\left(\varepsilon_{[\mathbf{j}]}-\varepsilon_{[\mathbf{q}]}\right) \gamma_{(1,0, \ldots, 0,0)}} P\left(\mathbf{J}_{(1,0, \ldots 0,0)}\right)}{\sqrt{\{\mathbf{j}\} !\{\mathbf{q}\} !}}\right. \\
& \left.\cdots, \frac{\mathbf{J}_{[\mathbf{k}]}^{(\mathbf{j}+\mathbf{q}) / 2} e^{-l\left(\varepsilon_{[\mathbf{j}]}-\varepsilon_{[\mathbf{q}]}\right) \gamma_{[\mathbf{k}]}} P\left(\mathbf{J}_{[\mathbf{k}]}\right)}{\sqrt{\{\mathbf{j}\} !\{\mathbf{q}\} !}}, \cdots, \frac{\mathbf{J}_{(1,1, \ldots, 1,1)}^{(\mathbf{j}+\mathbf{q}) / 2} e^{-l\left(\varepsilon_{[\mathbf{j}]}-\varepsilon_{[\mathbf{q}]}\right) \gamma_{(1,1, \ldots, 1,1)} P\left(\mathbf{J}_{(1,1, \ldots, 1,1)}\right)}}{\sqrt{\{\mathbf{j}\} !\{\mathbf{q}\} !}}\right) \\
& \times\left|\Psi_{[\mathbf{k}]} \otimes \Phi_{[\mathbf{j}]}^{[\mathbf{k}]}\right\rangle\left\langle\Psi_{[\mathbf{k}]} \otimes \Phi_{[\mathbf{q}]}^{[\mathbf{k}]}\right|,
\end{aligned}
$$

this latter being the density matrix representation in the NVCS basis. Then, (5.37) allows to derive the mean value of a diagonal operator $\mathscr{O}$ expressed in terms of $2^{M}$ operators, $\mathscr{O}(0,0, \ldots, 0, \ldots, 0,0), \mathscr{O}(1,0, \ldots, 0, \ldots, 0,0), \ldots, \mathscr{O}(1,1, \ldots, 1, \ldots, 1,1)$, acting on corresponding subspaces of the Hilbert space $\mathbb{C}^{2^{M}} \otimes \mathfrak{H}$.

Therefore, the density operator matrix elements can be expressed in this case as follows:

$$
\begin{aligned}
& \left\langle\Psi_{[\mathbf{k}]} \otimes \Phi_{[\mathbf{n}]}^{[\mathbf{k}]}|\hat{\rho}| \Psi_{[\mathbf{k}]} \otimes \Phi_{[[\mathbf{p}]}^{[\mathbf{k}]}\right\rangle \\
& =(2 \pi)^{N_{B}} \int_{0}^{\infty} \int_{0}^{\infty} \cdots \int_{0}^{\infty} d v_{(0, \cdots, 0,0)}\left(\mathbf{J}_{(0, \cdots, 0,0)}\right) \int_{0}^{\infty} \int_{0}^{\infty} \cdots \int_{0}^{\infty} d v_{(1, \cdots, 0,0)}\left(\mathbf{J}_{(1, \cdots, 0,0)}\right) \cdots \\
& \int_{0}^{\infty} \int_{0}^{\infty} \cdots \int_{0}^{\infty} d v_{[\mathbf{k}]}\left(\mathbf{J}_{[\mathbf{k}]}\right) \cdots \int_{0}^{\infty} \int_{0}^{\infty} \cdots \int_{0}^{\infty} d v_{(1, \cdots, 1,1)}\left(\mathbf{J}_{(1, \cdots, 1,1)}\right) \times \\
& \operatorname{diag}\left(\frac{\mathbf{J}_{(0,0, \ldots, 0,0)}^{(\mathbf{n}+\mathbf{p}) / 2}}{\sqrt{\{\mathbf{n}\} !\{\mathbf{p}\} !}} P\left(\mathbf{J}_{(0,0, \ldots, 0,0)}\right), \frac{\mathbf{J}_{(1,0, \ldots 0,0)}^{(\mathbf{n}+\mathbf{p}) / 2}}{\sqrt{\{\mathbf{n}\} !\{\mathbf{p}\} !}} P\left(\mathbf{J}_{(1,0, \ldots 0,0)}\right) \cdots, \frac{\mathbf{J}_{[\mathbf{k}]}^{(\mathbf{n}+\mathbf{p}) / 2}}{\sqrt{\{\mathbf{n}\} !\{\mathbf{p}\} !}} P\left(\mathbf{J}_{[\mathbf{k}]}\right), \cdots\right. \\
& \\
& \mathbf{J}_{(1,1, \ldots, 1,1)}^{(\mathbf{n}+\mathbf{p}) / 2} \\
& \left.\sqrt{\{\mathbf{n}\} !\{\mathbf{p}\} !} P\left(\mathbf{J}_{(1,1, \ldots, 1,1)}\right)\right) \delta_{[\mathbf{n}],[\mathbf{p}]}
\end{aligned}
$$

where the following identity 


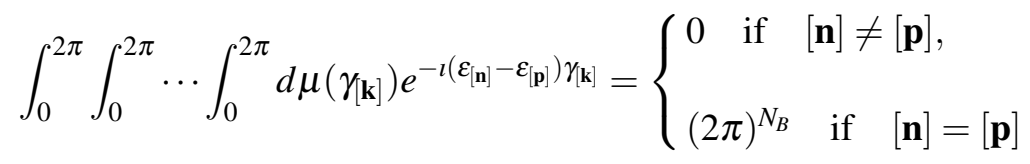

is used.

This is the matrix formulation of the density matrix in comparison with the quantity obtained in the case of bosonic states described in (5.26).

\section{NCS quantization of the complex plane}

Provided the resolution of the identity satisfied by the NCS, in this section we perform the NCS quantization of phase space classical observables. As a matter of illustration, the case of $q$-deformed CS is explicitly treated.

\subsection{General construction}

Such a quantization (see [13]) is realized through the mappings:

(i)

$$
\begin{aligned}
A_{\mathbf{z}}: \mathscr{D} \rightarrow \mathfrak{H}, \quad \mathbf{z} & \mapsto \int_{\mathscr{D}} \mathbf{z}\left|\eta_{\mathbf{z}}\right\rangle\left\langle\eta_{\mathbf{z}}\right| \mathscr{N}\left(|\mathbf{z}|^{2}\right) d \mu(\mathbf{z}, \overline{\mathbf{z}}) \\
& =\sum_{\mathbf{n}=0}^{\infty} \sqrt{(\mathbf{n}+1)} f(\mathbf{n}+1)\left|\Phi_{\mathbf{n}}^{[\mathbf{k}]}\right\rangle\left\langle\Phi_{\mathbf{n}+1}^{[\mathbf{k}]}\right|
\end{aligned}
$$

(ii)

$$
\begin{aligned}
A_{\overline{\mathbf{z}}}: \mathscr{D} \rightarrow \mathfrak{H}, \quad \mathbf{z} & \mapsto \int_{\mathscr{D}} \overline{\mathbf{z}}\left|\eta_{\mathbf{z}}\right\rangle\left\langle\eta_{\mathbf{z}}\right| \mathscr{N}\left(|\mathbf{z}|^{2}\right) d \mu(\mathbf{z}, \overline{\mathbf{z}}) \\
& =\sum_{\mathbf{n}=0}^{\infty} \sqrt{\mathbf{n}} f(\mathbf{n})\left|\Phi_{\mathbf{n}}^{[\mathbf{k}]}\right\rangle\left\langle\Phi_{\mathbf{n}-1}^{[\mathbf{k}]}\right|
\end{aligned}
$$

(iii)

$$
\begin{aligned}
A_{\mathbf{z}}^{\prime}: \tilde{\mathscr{D}} \rightarrow \mathfrak{H}, \quad \quad \mathbf{z} & \mapsto \int_{\mathscr{D}} \mathbf{z}\left|\eta_{\mathbf{z}}^{\prime}\right\rangle\left\langle\eta_{\mathbf{z}}^{\prime}\right| \mathscr{N}^{\prime}\left(|\mathbf{z}|^{2}\right) d \mu(\mathbf{z}, \overline{\mathbf{z}}) \\
& =\sum_{\mathbf{n}=0}^{\infty} \frac{\sqrt{(\mathbf{n}+1)}}{f(\mathbf{n}+1)}\left|\Phi_{\mathbf{n}}^{[\mathbf{k}]}\right\rangle\left\langle\Phi_{\mathbf{n}+1}^{[\mathbf{k}]}\right|,
\end{aligned}
$$

(iv)

$$
\begin{aligned}
A_{\overline{\mathbf{z}}}^{\prime}: \tilde{\mathscr{D}} \rightarrow \mathfrak{H}, \quad \quad \mathbf{z} & \mapsto \int_{\tilde{\mathscr{D}}} \overline{\mathbf{z}}\left|\eta_{\mathbf{z}}^{\prime}\right\rangle\left\langle\eta_{\mathbf{z}}^{\prime}\right| \mathscr{N}^{\prime}\left(|\mathbf{z}|^{2}\right) d \mu(\mathbf{z}, \overline{\mathbf{z}}) \\
& =\sum_{\mathbf{n}=0}^{\infty} \frac{\sqrt{\mathbf{n}}}{f(\mathbf{n})}\left|\Phi_{\mathbf{n}}^{[\mathbf{k}]}\right\rangle\left\langle\Phi_{\mathbf{n}-1}^{[\mathbf{k}]}\right| .
\end{aligned}
$$

The defined NCS can be used to calculate the expectation (mean) values of quantized elementary classical observables. For instance, the following mean values are computed: 


$$
\begin{aligned}
\left\langle\eta_{\mathbf{z}}\left|A_{\mathbf{z}}\right| \eta_{\mathbf{z}}\right\rangle=\mathbf{z}, & \left\langle\eta_{\mathbf{z}}\left|A_{\overline{\mathbf{z}}}\right| \eta_{\overline{\mathbf{z}}}\right\rangle=\overline{\mathbf{z}}, \quad\left\langle\eta_{\mathbf{z}}\left|A_{\mathbf{z}}^{2}\right| \eta_{\mathbf{z}}\right\rangle=\mathbf{z}^{2}, \quad\left\langle\eta_{\mathbf{z}}\left|A_{\overline{\mathbf{z}}}^{2}\right| \eta_{\mathbf{z}}\right\rangle=\overline{\mathbf{z}}^{2}, \\
\left\langle\eta_{\mathbf{z}}\left|A_{\overline{\mathbf{z}}} A_{\mathbf{z}}\right| \eta_{\mathbf{z}}\right\rangle=|\mathbf{z}|^{2} & \\
\left\langle\eta_{\mathbf{z}}\left|A_{\mathbf{z}} A_{\overline{\mathbf{z}}}\right| \eta_{\mathbf{z}}\right\rangle= & \left(\mathscr{N}\left(|\mathbf{z}|^{2}\right)\right)^{-1} \\
& \times\left[|\mathbf{z}|^{2} \sum_{\mathbf{n}=1}^{\infty} \frac{[f(\mathbf{n}+1)]^{2}}{[f(\mathbf{n})]^{2}} \frac{|\mathbf{z}|^{2(\mathbf{n}-1)}}{(\mathbf{n}-1) ![f(\mathbf{n}-1) !]^{2}}+\sum_{\mathbf{n}=0}^{\infty} \frac{[f(\mathbf{n}+1)]^{2}}{[f(\mathbf{n}) !]^{2}} \frac{|\mathbf{z}|^{2 \mathbf{n}}}{\mathbf{n} !}\right]
\end{aligned}
$$

such that for $f(\mathbf{n}) \rightarrow 1$ with $f(\mathbf{n}+1)=1=f(\mathbf{n}), f(\mathbf{n}+1) !=1=f(\mathbf{n})$ !, we get

$$
\left\langle\eta_{\mathbf{z}}\left|A_{\mathbf{z}} A_{\overline{\mathbf{z}}}\right| \eta_{\mathbf{z}}\right\rangle=|\mathbf{z}|^{2}+\left\langle\eta_{\mathbf{z}}|\mathbb{I}| \eta_{\mathbf{z}}\right\rangle
$$

as in the case of the standard CS. Moreover, from the quantities:

$$
\begin{aligned}
A_{|\mathbf{z}|^{2}} & =\sum_{\mathbf{n}=0}^{\infty}(\mathbf{n}+1)[f(\mathbf{n}+1)]^{2}\left|\Phi_{\mathbf{n}}^{[\mathbf{k}]}\right\rangle\left\langle\Phi_{\mathbf{n}}^{[\mathbf{k}]}\right|, \\
A_{\mathbf{z}^{2}} & =\sum_{\mathbf{n}=0}^{\infty} \sqrt{(\mathbf{n}+2)(\mathbf{n}+1)} f(\mathbf{n}+2) f(\mathbf{n}+1)\left|\Phi_{\mathbf{n}}^{[\mathbf{k}]}\right\rangle\left\langle\Phi_{\mathbf{n}+2}^{[\mathbf{k}]}\right|, \\
A_{\overline{\mathbf{z}}^{2}} & =\sum_{\mathbf{n}=0}^{\infty} \sqrt{(\mathbf{n}+2)(\mathbf{n}+1)} f(\mathbf{n}+2) f(\mathbf{n}+1)\left|\Phi_{\mathbf{n}+2}^{[\mathbf{k}]}\right\rangle\left\langle\Phi_{\mathbf{n}}^{[\mathbf{k}]}\right|,
\end{aligned}
$$

we derive the commutators:

$$
\begin{aligned}
{\left[A_{|\mathbf{z}|^{2}}, A_{\mathbf{z}}\right]=} & \sum_{\mathbf{n}=0}^{\infty} \sqrt{(\mathbf{n}+1)} f(\mathbf{n}+1) \\
& \times\left\{(\mathbf{n}+1)[f(\mathbf{n}+1)]^{2}-(\mathbf{n}+2)[f(\mathbf{n}+2)]^{2}\right\}\left|\Phi_{\mathbf{n}}^{[\mathbf{k}]}\right\rangle\left\langle\Phi_{\mathbf{n}+1}^{[\mathbf{k}]}\right|
\end{aligned}
$$

$$
\begin{aligned}
{\left[A_{|\mathbf{z}|^{2}}, A_{\overline{\mathbf{z}}}\right]=} & \sum_{\mathbf{n}=0}^{\infty} \sqrt{(\mathbf{n}+1)} f(\mathbf{n}+1) \\
& \times\left\{(\mathbf{n}+2)[f(\mathbf{n}+2)]^{2}-(\mathbf{n}+1)[f(\mathbf{n}+1)]^{2}\right\}\left|\Phi_{\mathbf{n}+1}^{[\mathbf{k}]}\right\rangle\left\langle\Phi_{\mathbf{n}}^{[\mathbf{k}]}\right|
\end{aligned}
$$

$$
\begin{aligned}
{\left[A_{|\mathbf{z}|^{2}}, A_{\mathbf{z}^{2}}\right]=} & \sqrt{(\mathbf{n}+2)(\mathbf{n}+1)} f(\mathbf{n}+2) f(\mathbf{n}+1) \\
& \times\left\{(\mathbf{n}+1)[f(\mathbf{n}+1)]^{2}-(\mathbf{n}+3)[f(\mathbf{n}+3)]^{2}\right\}\left|\Phi_{\mathbf{n}}^{[\mathbf{k}]}\right\rangle\left\langle\Phi_{\mathbf{n}+2}^{[\mathbf{k}]}\right|
\end{aligned}
$$




$$
\begin{aligned}
{\left[A_{|\mathbf{z}|^{2}}, A_{\overline{\mathbf{z}}^{2}}\right]=} & \sqrt{(\mathbf{n}+2)(\mathbf{n}+1)} f(\mathbf{n}+2) f(\mathbf{n}+1) \\
& \times\left\{(\mathbf{n}+3)[f(\mathbf{n}+3)]^{2}-(\mathbf{n}+1)[f(\mathbf{n}+1)]^{2}\right\}\left|\Phi_{\mathbf{n}+2}^{[\mathbf{k}]}\right\rangle\left\langle\Phi_{\mathbf{n}}^{[\mathbf{k}]}\right| .
\end{aligned}
$$

Considering the usual phase space conjugate coordinates $(\mathbf{q}, \mathbf{p})$ through $\mathbf{z}=\frac{\mathbf{q}+i \mathbf{p}}{\sqrt{2}}$, we get for the classical position function $\mathbf{q}$ :

$$
Q:=A_{\mathbf{q}}=\frac{1}{\sqrt{2}}\left(A_{\mathbf{z}}+A_{\overline{\mathbf{z}}}\right),
$$

and for the classical momentum function $\mathbf{p}$ :

$$
P:=A_{\mathbf{p}}=\frac{1}{i \sqrt{2}}\left(A_{\mathbf{z}}-A_{\overline{\mathbf{z}}}\right)
$$

The mean values of the operators $Q, P$ and $Q^{2}, P^{2}$ can be written as follows:

$$
\begin{aligned}
\left\langle\eta_{\mathbf{z}}|Q| \eta_{\mathbf{z}}\right\rangle & =\frac{1}{\sqrt{2}}\left[\left\langle\eta_{\mathbf{z}}\left|A_{\mathbf{z}}\right| \eta_{\mathbf{z}}\right\rangle+\left\langle\eta_{\mathbf{z}}\left|A_{\overline{\mathbf{z}}}\right| \eta_{\mathbf{z}}\right\rangle\right]=\frac{1}{\sqrt{2}}(\mathbf{z}+\overline{\mathbf{z}})=\mathbf{q} \\
\left\langle\eta_{\mathbf{z}}|P| \eta_{\mathbf{z}}\right\rangle & =\frac{1}{i \sqrt{2}}\left[\left\langle\eta_{\mathbf{z}}\left|A_{\mathbf{z}}\right| \eta_{\mathbf{z}}\right\rangle-\left\langle\eta_{\mathbf{z}}\left|A_{\overline{\mathbf{z}}}\right| \eta_{\mathbf{z}}\right\rangle\right]=\frac{1}{i \sqrt{2}}(\mathbf{z}-\overline{\mathbf{z}})=\mathbf{p} \\
\left\langle\eta_{\mathbf{z}}\left|Q^{2}\right| \eta_{\mathbf{z}}\right\rangle & =\frac{1}{2}\left[\left\langle\eta_{\mathbf{z}}\left|A_{\mathbf{z}}^{2}\right| \eta_{\mathbf{z}}\right\rangle+\left\langle\eta_{\mathbf{z}}\left|A_{\overline{\mathbf{z}}}^{2}\right| \eta_{\mathbf{z}}\right\rangle+\left\langle\eta_{\mathbf{z}}\left|\left\{A_{\mathbf{z}}, A_{\overline{\mathbf{z}}}\right\}\right| \eta_{\mathbf{z}}\right\rangle\right] \\
\left\langle\eta_{\mathbf{z}}\left|P^{2}\right| \eta_{\mathbf{z}}\right\rangle & =-\frac{1}{2}\left[\left\langle\eta_{\mathbf{z}}\left|A_{\mathbf{z}}^{2}\right| \eta_{\mathbf{z}}\right\rangle+\left\langle\eta_{\mathbf{z}}\left|A_{\overline{\mathbf{z}}}^{2}\right| \eta_{\mathbf{z}}\right\rangle-\left\langle\eta_{\mathbf{z}}\left|\left\{A_{\mathbf{z}}, A_{\overline{\mathbf{z}}}\right\}\right| \eta_{\mathbf{z}}\right\rangle\right]
\end{aligned}
$$

with the anti-commutator of two operators $\mathscr{A}$ and $\mathscr{B}$ given by $\{\mathscr{A}, \mathscr{B}\}:=\mathscr{A} \mathscr{B}+\mathscr{B} \mathscr{A}$, where

$$
\begin{aligned}
\left\langle\eta_{\mathbf{z}}\left|\left\{A_{\mathbf{z}}, A_{\overline{\mathbf{z}}}\right\}\right| \eta_{\mathbf{z}}\right\rangle= & \left(\mathscr{N}\left(|\mathbf{z}|^{2}\right)\right)^{-1} \\
& \times\left[\sum_{\mathbf{m}=0}^{\infty}\left\{(\mathbf{m}+1)[f(\mathbf{m}+1)]^{2}+\mathbf{m}[f(\mathbf{m})]^{2}\right\} \times \frac{|\mathbf{z}|^{2 \mathbf{m}}}{\{\mathbf{m}\} !} .\right.
\end{aligned}
$$

Using (6.5)-(6.7), the expressions (6.17) are obtained as

$$
\begin{gathered}
\left\langle\eta_{\mathbf{z}}\left|Q^{2}\right| \eta_{\mathbf{z}}\right\rangle=\frac{1}{2}\left[\mathbf{q}^{2}-\mathbf{p}^{2}+\left\langle\eta_{\mathbf{z}}\left|\left\{A_{\mathbf{z}}, A_{\overline{\mathbf{z}}}\right\}\right| \eta_{\mathbf{z}}\right\rangle\right] \\
\left\langle\eta_{\mathbf{z}}\left|P^{2}\right| \eta_{\mathbf{z}}\right\rangle=-\frac{1}{2}\left[\mathbf{q}^{2}-\mathbf{p}^{2}-\left\langle\eta_{\mathbf{z}}\left|\left\{A_{\mathbf{z}}, A_{\overline{\mathbf{z}}}\right\}\right| \eta_{\mathbf{z}}\right\rangle\right]
\end{gathered}
$$

with $\left\langle\eta_{\mathbf{z}}\left|\left\{A_{\mathbf{z}}, A_{\overline{\mathbf{z}}}\right\}\right| \eta_{\mathbf{z}}\right\rangle$ given in (6.18).

Then, with $\{\mathbf{m}\}:=\mathbf{m}[f(\mathbf{m})]^{2}$, we get the dispersions: 


$$
\begin{aligned}
(\Delta Q)^{2} & =\left\langle Q^{2}\right\rangle-(\langle Q\rangle)^{2}=-|\mathbf{z}|^{2}+\frac{1}{2}\left(\mathscr{N}\left(|\mathbf{z}|^{2}\right)\right)^{-1}\left[\sum_{\mathbf{m}=0}^{\infty}[\{\mathbf{m}+1\}+\{\mathbf{m}\}] \times \frac{|\mathbf{z}|^{2 \mathbf{m}}}{\{\mathbf{m}\} !}\right] \\
& =-\frac{1}{2}|\mathbf{z}|^{2}+\mathscr{G}\left(|\mathbf{z}|^{2}\right) \\
(\Delta P)^{2} & =\left\langle P^{2}\right\rangle-(\langle P\rangle)^{2}=-|\mathbf{z}|^{2}+\frac{1}{2}\left(\mathscr{N}\left(|\mathbf{z}|^{2}\right)\right)^{-1}\left[\sum_{\mathbf{m}=0}^{\infty}[\{\mathbf{m}+1\}+\{\mathbf{m}\}] \times \frac{|\mathbf{z}|^{2 \mathbf{m}}}{\{\mathbf{m}\} !}\right] \\
& =-\frac{1}{2}|\mathbf{z}|^{2}+\mathscr{G}\left(|\mathbf{z}|^{2}\right)
\end{aligned}
$$

where

$$
\mathscr{G}\left(|\mathbf{z}|^{2}\right):=\frac{1}{2}\left(\mathscr{N}\left(|\mathbf{z}|^{2}\right)\right)^{-1}\left[\sum_{\mathbf{m}=0}^{\infty}\{\mathbf{m}+1\} \times \frac{|\mathbf{z}|^{2 \mathbf{m}}}{\{\mathbf{m}\} !}\right] .
$$

Now, taking into account both (6.14) and (6.15), and by defining $\langle[Q, P]\rangle:=\left\langle\eta_{\mathbf{z}}|[Q, P]| \eta_{\mathbf{z}}\right\rangle$, we get

$$
\langle[Q, P]\rangle=i\left(\mathscr{N}\left(|\mathbf{z}|^{2}\right)\right)^{-1}\left[\sum_{\mathbf{m}=0}^{\infty}[\{\mathbf{m}+1\}-\{\mathbf{m}\}] \times \frac{|\mathbf{z}|^{2 \mathbf{m}}}{\{\mathbf{m}\} !}\right]
$$

which leads, by using (6.21)-(6.23), to

$$
(\Delta Q)^{2}=(\Delta P)^{2}=\frac{1}{2}|\langle[Q, P]\rangle|
$$

and, therefore, to the relation

$$
(\Delta Q)^{2}(\Delta P)^{2}=\frac{1}{4}|\langle[Q, P]\rangle|^{2}
$$

attesting that the NCS $\left|\eta_{\mathbf{z}}\right\rangle$ are intelligent, as in the standard situation, for the operators $Q$ and $P$ obtained from the CS quantization of the classical observables $\mathbf{q}$ and $\mathbf{p}$, respectively.

\subsection{Illustration: case of Quesne's q-deformed CS [27]}

The Quesne's $q$-deformed CS [27] can be retrieved from the above generalization by setting $f(\mathbf{n})=$ $\sqrt{\frac{[\mathbf{n}]_{q}}{\mathbf{n}}}$, with $0<q<1$ and $\mathbf{z} \in \mathbb{C}$, and

$$
\left|\eta_{\mathbf{z}}\right\rangle_{q}=\left(\mathscr{N}_{q}\left(|\mathbf{z}|^{2}\right)\right)^{-1 / 2} \sum_{\mathbf{n}=0}^{\infty} \frac{\mathbf{z}^{\mathbf{n}}}{\sqrt{[\mathbf{n}]_{q} !}}\left|\Phi_{\mathbf{n}}^{[\mathbf{k}]}\right\rangle_{q}, \quad \mathscr{N}_{q}\left(|\mathbf{z}|^{2}\right)=\sum_{\mathbf{n}=0}^{\infty} \frac{|\mathbf{z}|^{2 \mathbf{n}}}{[\mathbf{n}]_{q} !}=E_{q}\left(|\mathbf{z}|^{2}\right)
$$

where the $q$-factorial 


$$
[\mathbf{n}]_{q} ! \equiv \begin{cases}1 & \text { if } \quad \mathbf{n}=0 \\ {[\mathbf{n}]_{q}[\mathbf{n}-1]_{q} \ldots[1]_{q}} & \text { if } \quad \mathbf{n}=1,2,3, \ldots\end{cases}
$$

is defined in terms of the $q$-numbers

$$
[\mathbf{n}]_{q} \equiv \frac{q^{\mathbf{n}}-1}{q-1}=1+q+q^{2}+\cdots+q^{\mathbf{n}-1}
$$

such that in the limit $q \rightarrow 1,[\mathbf{n}]_{q},[\mathbf{n}]_{q}$ ! tend to $\mathbf{n}$ and $\mathbf{n}$ !, respectively.

In this case the deformation (2.3), with $f_{l}\left(N_{l}\right)=\sqrt{\frac{\left[N_{l}\right]_{q}}{N_{l}}}$, is obtained through the following correspondences:

$$
a_{l} \rightarrow b_{l}=a_{l} \sqrt{\frac{\left[N_{l}\right]_{q}}{N_{l}}}, \quad a_{l}^{\dagger} \rightarrow b_{l}^{\dagger}=\sqrt{\frac{\left[N_{l}\right]_{q}}{N_{l}}} a_{l}^{\dagger}, \quad 1 \leq l \leq N_{B}
$$

where $N_{l}=a_{l}^{\dagger} a_{l}$ and $\left[N_{l}\right]_{q}$ defined as in (6.29). From (6.30), it comes that $b_{l}^{\dagger} b_{l}=\left[N_{l}\right]_{q}$ and $b_{l} b_{l}^{\dagger}=\left[N_{l}+1\right]_{q}$. The actions of the operators $b_{l}, b_{l}^{\dagger}$ on the states $\left|\Phi_{n_{l}}^{[\mathbf{k}]}\right\rangle_{q}$ are given for $1 \leq l \leq N_{B}$ by

$$
b_{l}\left|\Phi_{n_{l}}^{[\mathbf{k}]}\right\rangle_{q}=\sqrt{\left[n_{l}\right]_{q}}\left|\Phi_{n_{l}-1}^{[\mathbf{k}]}\right\rangle_{q}, \quad b_{l}^{\dagger}\left|\Phi_{n_{l}}^{[\mathbf{k}]}\right\rangle_{q}=\sqrt{\left[n_{l}+1\right]_{q}}\left|\Phi_{n_{l}+1}^{[\mathbf{k}]}\right\rangle_{q}, \quad b_{l} b_{l}^{\dagger}-q b_{l}^{\dagger} b_{l}=\mathbb{I}
$$

where in the limit $q \rightarrow 1,\left[b_{l}, b_{l}^{\dagger}\right]=\mathbb{I}$.

The states $\left|\Phi_{\mathbf{n}}^{[\mathbf{k}]}\right\rangle_{q}=\bigotimes_{l=1}^{N_{B}}\left|\Phi_{n_{l}}^{[\mathbf{k}]}\right\rangle_{q}$ are such that the states $\left|\Phi_{n_{l}}^{[\mathbf{k}]}\right\rangle_{q}$ are obtained with (2.34) as

$$
\left|\Phi_{n_{l}}^{[\mathbf{k}]_{l}}\right\rangle_{q}=\frac{\left(\mathscr{A}_{[\mathbf{k}]}^{\dagger}\right)^{n_{l}}}{\sqrt{\left[n_{l}\right]_{q} !}}\left|\Phi_{0_{l}}^{[\mathbf{k}]}\right\rangle_{q}, \quad \mathscr{A}_{[\mathbf{k}]_{l}}=e^{i \sqrt{2} \frac{\left.g_{[\mathbf{k}}\right]}{\omega_{l}} \mathscr{P}_{l}} b_{l} e^{-i \sqrt{2} \frac{\left.g^{g} \mathbf{k}\right]}{\omega_{l}} \mathscr{P}_{l}}
$$

with $\left|\Phi_{0_{l}}^{[\mathbf{k}]}\right\rangle_{q}=e^{i \sqrt{2} \frac{\left.g_{[} \mathbf{k}\right]}{\omega_{l}} \mathscr{P}_{l}}|0\rangle_{q}$, where we assume $|0\rangle_{q}=|0\rangle,\left(b_{l}|0\rangle_{q}=0\right)$, and $\mathscr{P}_{l}$ with $f_{l}\left(N_{l}\right)=$ $\sqrt{\frac{\left[N_{l}\right]_{q}}{N_{l}}}$ is given as in (2.33). The $\left|\Phi_{\mathbf{n}}^{[\mathbf{k}]}\right\rangle_{q}$ are identified with the states $|n\rangle_{q}$ of the Hilbert space $\mathscr{F}_{q}$ [27] which is the $q$-deformed Fock space associated with the $q$-boson creation and annihilation operators.

Provided the resolution of the identity on $\mathscr{F}_{q}$ spanned by the states $\left|\Phi_{\mathbf{n}}^{[\mathbf{k}]}\right\rangle_{q}$

$$
\iint_{\mathbb{C}} d^{2} \mathbf{z}\left|\eta_{\mathbf{z}}\right\rangle_{q} W_{q}\left(|\mathbf{z}|^{2}\right)_{q}\left\langle\eta_{\mathbf{z}}\left|=\sum_{\mathbf{n}=0}^{\infty}\right| \Phi_{\mathbf{n}}^{[\mathbf{k}]}\right\rangle_{q q}\left\langle\Phi_{\mathbf{n}}^{[\mathbf{k}]}\right|=I_{\mathscr{F}_{q}}
$$

where

$$
W_{q}\left(|\mathbf{z}|^{2}\right)=\frac{q-1}{\pi \ln q} \frac{E_{q}\left(|\mathbf{z}|^{2}\right)}{E_{q}\left(q|\mathbf{z}|^{2}\right)},
$$

the CS quantization is performed as in (6.1)-(6.2) and leads to the $q$-deformed analogue of (6.18): 


$$
{ }_{q}\left\langle\eta_{\mathbf{z}}\left|\left\{A_{\mathbf{z}}, A_{\overline{\mathbf{z}}}\right\}\right| \eta_{\mathbf{z}}\right\rangle_{q}=\left(\mathscr{N}_{q}\left(|\mathbf{z}|^{2}\right)\right)^{-1}\left[\sum_{\mathbf{m}=0}^{\infty}\left\{[\mathbf{m}+1]_{q}+[\mathbf{m}]_{q}\right\} \times \frac{|\mathbf{z}|^{2 \mathbf{m}}}{[\mathbf{m}]_{q} !}\right] .
$$

The dispersions are given by

$$
(\Delta Q)_{q}^{2}=(\Delta P)_{q}^{2}=-\frac{1}{2}|\mathbf{z}|^{2}+\mathscr{G}_{q}\left(|\mathbf{z}|^{2}\right)
$$

where

$$
\mathscr{G}_{q}\left(|\mathbf{z}|^{2}\right):=\frac{1}{2}\left(\mathscr{N}_{q}\left(|\mathbf{z}|^{2}\right)\right)^{-1}\left[\sum_{\mathbf{m}=0}^{\infty}[\mathbf{m}+1]_{q} \frac{|\mathbf{z}|^{2 \mathbf{m}}}{[\mathbf{m}]_{q} !}\right] .
$$

Proceeding as in (6.21)-(6.25) with (6.36) and (6.37), we arrive at

$$
(\Delta Q)_{q}^{2}(\Delta P)_{q}^{2}=\frac{1}{4}\left|\left\langle[Q, P]_{q}\right\rangle\right|^{2}
$$

\section{Quantum optical features of the NCS}

Now, we exploit the results issued from the above developed quantization procedure to inspect some quantum optical properties of the constructed NCS. Roy et al [28] pointed out interesting properties exhibited by NCS, such as squeezing and sub-Poissonian behavior. For more details see also [12]. In the following, we discuss some other relevant aspects like the signal-to-quantum-noise ratio and the Mandel parameter.

\subsection{Signal-to-quantum-noise ratio (SNR)}

Signal-to-quantum-noise ratio (SNR) is relevant when studying, for example, the exciton spin relaxation for dynamics of photoexcited excitons in an ensemble of InAs/GaAs self-assembled quantum dots [23]. For a normalized state $|\phi\rangle$, in terms of the self adjoint quadrature operator $Q$, the SNR is defined as [25]

$$
\sigma_{|\phi\rangle}=\frac{\langle Q\rangle_{|\phi\rangle}^{2}}{(\Delta Q)_{|\phi\rangle}^{2}}
$$

In the NCS $\left|\eta_{\mathbf{z}}\right\rangle$, we obtain

$$
\sigma_{\mathbf{z}}=\frac{\mathbf{q}^{2}}{(\Delta Q)^{2}}=\frac{2|\mathbf{z}|^{2} \cos ^{2} \phi}{-\frac{1}{2}|\mathbf{z}|^{2}+\mathscr{G}\left(|\mathbf{z}|^{2}\right)}
$$

In the case where $f(\mathbf{n}) \rightarrow 1$ with $f(\mathbf{n}+1)=1=f(\mathbf{n}), f(\mathbf{n}+1) !=1=f(\mathbf{n})$ !, we have $\mathscr{G}\left(|\mathbf{z}|^{2}\right)=$ $\frac{1}{2}+\frac{1}{2}|\mathbf{z}|^{2}$ providing $\sigma_{\mathbf{z}}=4|\mathbf{z}|^{2} \cos ^{2} \phi$, this latter being the SNR for the canonical CS (CCS).

For the $q$-deformed CS, the SNR is given by 


$$
\sigma_{\mathbf{z}_{q}}=\frac{\mathbf{q}^{2}}{(\Delta Q)_{q}^{2}}=\frac{2|\mathbf{z}|^{2} \cos ^{2} \phi}{-\frac{1}{2}|\mathbf{z}|^{2}+\mathscr{G}_{q}\left(|\mathbf{z}|^{2}\right)}
$$

such that in the limit $q \rightarrow 1, \mathscr{G}_{q}\left(|\mathbf{z}|^{2}\right) \rightarrow \frac{1}{2}+\frac{1}{2}|\mathbf{z}|^{2}$ leading to $\sigma_{\mathbf{z}_{q}} \rightarrow 4|\mathbf{z}|^{2} \cos ^{2} \phi$.

\subsection{Mandel parameter}

The Mandel parameter known as a convenient noise-indicator of a non-classical field and defined by [19]

$$
\mathscr{Q}=\frac{(\Delta N)^{2}}{\langle N\rangle}-1 \equiv \mathscr{F}-1
$$

is closely related to the normalized variance also called the quantum Fano factor $\mathscr{F}$ [6], given by $\mathscr{F}=(\Delta N)^{2} /\langle N\rangle$, of the photon distribution. For $\mathscr{F}<1(\mathscr{Q} \leq 0)$, the emitted light is referred to as sub-Poissonian, with $(\mathscr{F}=1 ; \mathscr{Q}=0)$, whereas for $\mathscr{F}>1,(\mathscr{Q}>0)$ the light is called superPoissonian.

We get for $\mathscr{N}=A^{\dagger} A$, where $A$ and $A^{\dagger}$ are given as in (2.32) with $A\left|\Phi_{\mathbf{n}}^{[\mathbf{k}]}\right\rangle=\sqrt{\{\mathbf{n}\}}\left|\Phi_{\mathbf{n}-1}^{[\mathbf{k}]}\right\rangle$ and $A^{\dagger}\left|\Phi_{\mathbf{n}}^{[\mathbf{k}]}\right\rangle=\sqrt{\{\mathbf{n}+1\}}\left|\Phi_{\mathbf{n}+\mathbf{1}}^{[\mathbf{k}]}\right\rangle$, the following mean values

$$
\left\langle\eta_{\mathbf{z}}|\mathscr{N}| \eta_{\mathbf{z}}\right\rangle=|\mathbf{z}|^{2}\left(\mathscr{N}\left(|\mathbf{z}|^{2}\right)\right)^{-1} \sum_{\mathbf{n}=1}^{\infty} \frac{|\mathbf{z}|^{2(\mathbf{n}-1)}}{\{(\mathbf{n}-1)\} !}=|\mathbf{z}|^{2}
$$

and

$$
\left\langle\eta_{\mathbf{z}}\left|\mathscr{N}^{2}\right| \eta_{\mathbf{z}}\right\rangle=\left(\mathscr{N}\left(|\mathbf{z}|^{2}\right)\right)^{-1} \sum_{\mathbf{n}=0}^{\infty} \frac{|\mathbf{z}|^{2 \mathbf{n}}}{\{\mathbf{n}\} !}\{\mathbf{n}\}^{2} .
$$

Thus, the dispersion is derived in the NCS $\left|\eta_{\mathbf{z}}\right\rangle$ as follows:

$$
\begin{aligned}
(\Delta \mathscr{N})_{\mathbf{z}}^{2} & =\left\langle\mathscr{N}^{2}\right\rangle_{\mathbf{z}}-\left(\langle\mathscr{N}\rangle_{\mathbf{z}}\right)^{2} \\
& =\left(\mathscr{N}\left(|\mathbf{z}|^{2}\right)\right)^{-1} \sum_{\mathbf{n}=0}^{\infty} \frac{|\mathbf{z}|^{2 \mathbf{n}}}{\{\mathbf{n}\} !}\{\mathbf{n}\}^{2}-\left(|\mathbf{z}|^{2}\right)^{2} .
\end{aligned}
$$

The Mandel parameter is therefore provided for a given $f$-deformed function of the number operator in the manner

$$
\begin{aligned}
\mathscr{Q} & =\frac{(\Delta \mathscr{N})_{\mathbf{z}}^{2}}{\langle\mathscr{N}\rangle_{\mathbf{z}}}-1 \\
& =\left(\mathscr{N}\left(|\mathbf{z}|^{2}\right)\right)^{-1} \sum_{\mathbf{n}=0}^{\infty} \frac{|\mathbf{z}|^{2 \mathbf{n}}}{\{\mathbf{n}\} !}\{\mathbf{n}+1\}-\left(|\mathbf{z}|^{2}+1\right) .
\end{aligned}
$$

In the case where $f(\mathbf{n}) \rightarrow 1$ with $f(\mathbf{n}+1)=1=f(\mathbf{n}), f(\mathbf{n}+1) !=1=f(\mathbf{n})$ !, we have the quantities related to the canonical coherent states (CCS) given by 


$$
\left\langle N^{2}\right\rangle_{\mathbf{z}}=|\mathbf{z}|^{4}+|\mathbf{z}|^{2}, \quad\langle N\rangle_{\mathbf{z}}=|\mathbf{z}|^{2}
$$

leading to the following Mandel parameter related to a Poissonian statistics:

$$
\begin{aligned}
\mathscr{Q}_{\mathrm{ccs}} & =\frac{\left(|\mathbf{z}|^{4}+|\mathbf{z}|^{2}\right)-\left(|\mathbf{z}|^{2}\right)^{2}}{|\mathbf{z}|^{2}}-1 \\
& =0 .
\end{aligned}
$$

For the CS $\left|\eta_{\mathbf{z}}\right\rangle_{q}$, the Mandel parameter denoted by $\mathscr{Q}_{q}$ is obtained as

$$
\begin{aligned}
\mathscr{Q}_{q} & =\frac{\left(\mathscr{N}_{q}\left(|\mathbf{z}|^{2}\right)\right)^{-1} \sum_{\mathbf{n}=0}^{\infty} \frac{|\mathbf{z}|^{2 \mathbf{n}}}{[\mathbf{n}]_{q} !}[\mathbf{n}]_{q}^{2}-\left(|\mathbf{z}|^{2}\right)^{2}}{|\mathbf{z}|^{2}}-1 \\
& =\left(\mathscr{N}_{q}\left(|\mathbf{z}|^{2}\right)\right)^{-1} \sum_{\mathbf{n}=0}^{\infty} \frac{|\mathbf{z}|^{2 \mathbf{n}}}{[\mathbf{n}]_{q} !}[\mathbf{n}+1]_{q}-\left(|\mathbf{z}|^{2}+1\right)
\end{aligned}
$$

such that in the limit $q \rightarrow 1$ we get $\mathscr{Q}_{q} \rightarrow \mathscr{Q}_{\text {ccs }}=0$.

Note that in [14], the quantum statistical properties of the deformed states are discussed in the context of conventional as well as deformed quantum optics.

Introducing the $f$-deformed $S U_{f}(1,1)$ algebra (see [18] and references therein), which consists of three generators

$$
\mathscr{K}_{-}=A_{l} A_{k}, \quad \mathscr{K}_{+}=A_{l}^{\prime \dagger} A_{k}^{\prime \dagger}, \quad \mathscr{K}_{0}=\frac{1}{2}\left(\mathscr{N}_{l}+\mathscr{N}_{k}+\mathbb{I}\right), \quad \mathscr{N}_{l}=A_{l}^{\prime \dagger} A_{l} \quad \text { and } \quad \mathscr{N}_{k}=A_{k}^{\prime \dagger} A_{k}
$$

where $A_{l}, A_{k}$ and $A_{l}^{\prime \dagger}, A_{k}^{\prime \dagger}\left(1 \leq k, l \leq N_{B}\right)$ are given by (2.30) and (2.31), we get the following commutation relations

$$
\left[\mathscr{K}_{+}, \mathscr{K}_{-}\right]=-2 \mathscr{K}_{0}, \quad\left[\mathscr{K}_{0}, \mathscr{K}_{ \pm}\right]= \pm \mathscr{K}_{ \pm}
$$

This algebra is a generalization of the $S U(1,1)$ Lie algebra [26]. Indeed, when $\mathscr{K}_{+}$and $\mathscr{K}_{-}$are Hermitian conjugate to each other in the special case of $f\left(N_{i}\right)=1$, i.e., $\mathscr{K}_{-}^{\dagger}=\mathscr{K}_{+}^{\dagger}$, the $S U_{f}(1,1)$ algebra contracts to the $S U(1,1)$ Lie algebra.

Let now

$$
X=\frac{\mathscr{K}_{-}^{\dagger}+\mathscr{K}_{-}}{2}, \quad Y=\frac{i\left(\mathscr{K}_{-}^{\dagger}-\mathscr{K}_{-}\right)}{2}
$$

be the $f$-deformed quadrature operators satisfying the commutation relation

$$
[X, Y]=\frac{i}{2}\left[\mathscr{K}_{-}, \mathscr{K}_{-}^{\dagger}\right]=\frac{i}{2}\left[\left(N_{k}+1\right) f_{k}^{2}\left(N_{k}+1\right)\left(N_{l}+1\right) f_{l}^{2}\left(N_{l}+1\right)-N_{k} f_{k}^{2}\left(N_{k}\right) N_{l} f_{l}^{2}\left(N_{l}\right)\right] .
$$

The following uncertainty relation 


$$
\left\langle(\Delta X)^{2}\right\rangle\left\langle(\Delta Y)^{2}\right\rangle \geq \frac{1}{16}\left|\left\langle\left[\mathscr{K}_{-}, \mathscr{K}_{-}^{\dagger}\right]\right\rangle\right|^{2}
$$

holds and the $S U_{f}(1,1)$ squeezing is provided by the relation:

$$
\left\langle\left(\Delta X_{k}\right)^{2}\right\rangle<\frac{1}{4}\left|\left\langle\left[\mathscr{K}_{-}, \mathscr{K}_{-}^{\dagger}\right]\right\rangle\right|, \quad X_{k}=X, Y
$$

\section{Concluding remarks}

In this work we have provided a construction of a dual pair of nonlinear coherent states (NCS) in the context of changes of bases in the underlying Hilbert space for a Hamiltonian describing the electron-phonon dynamics in condensed matter physics, which obeys a $f$-deformed Heisenberg algebra. The existence and properties of reproducing kernel in the NCS Hilbert space have been studied and discussed; the probability density and its dynamics in the basis of constructed CS have been analyzed. Moreover, a NCS quantization of classical phase space observables has been performed and illustrated in the case of the Quesne's $q$-deformed CS corresponding to the situation where the deformation structure function $f(N)=\sqrt{\frac{[N]_{q}}{N}}$.

Another important result in this work consists in the generalization, in a NVCS basis, of the Glauber-Sudarshan $P$-representation of the density matrix for a diagonal operator, to complex systems such as electron-phonon models in condensed matter physics. The same approach can be applied to other quantum optical models including spin-orbit interactions systems [31] and the Jaynes-Cummings model [17]. The three main axioms characterizing the reproducing kernel property of the density matrix have been highlighted in the NCS. Besides, the analysis of the performed NCS quantization has permitted to investigate the signal-to-quantum-noise ratio and Mandel parameter measuring the deviation of a Poisson-like distribution from the Poisson distribution, pertaining to the considered deformed algebra. It is shown that the NCS also exhibit squeezing property under the $S U_{f}(1,1)$ algebra built from the constructed deformed operator algebra.

\section{Acknowledgements}

The authors are grateful to the referee for his useful comments which helped them to improve the paper. This work is partially supported by the Abdus Salam International Centre for Theoretical Physics (ICTP, Trieste, Italy) through the Office of External Activities (OEA) - Prj-15. The ICMPA is in partnership with the Daniel Iagolnitzer Foundation (DIF), France.

\section{References}

[1] S. T. Ali, J. P. Antoine and J. P. Gazeau, Coherent States, Wavelets and their Generalizations (SpringerVerlag, New York, Berlin, Heidelberg, 2000; 2nd ed. 2013).

[2] S. T. Ali, M. Engliš and J. P. Gazeau, Vector coherent states from Plancherel's theorem, Clifford algebras and matrix domains, J. Phys. A: Math. Gen. 37 (2004) 6067-6089.

[3] S. T. Ali, R. Roknizadeh and M. Tavassoly, Representations of coherent states in non-orthogonal bases, J. Phys. A: Math. Gen. 37 (2004) 4407-4422.

[4] I. Aremua and M. N. Hounkonnou, Vector coherent states for nanoparticle systems, J. Phys. A: Math. Theor. 44 (2011) 465208. 
[5] I. Aremua, M. N. Hounkonnou and E. Baloïtcha, Coherent states for Landau levels: algebraic and thermodynamical properties, preprint (2013), arXiv: math-ph/1301.6280.

[6] J. Bajer and A. Miranowicz, Sub-Poissonian photon statistics of higher harmonics: quantum predictions via classical trajectories, J. Opt. B: Quantum Semiclass. Opt. 2 (2000) L10.

[7] J. Ben Geloun and M. N. Hounkonnou, Canonical and nonlinear vector coherent states of generalized models with spin-orbit interaction, J. Math. Phys. 48 (2007) 093505;

J. Ben Geloun and M. N. Hounkonnou, New classes of nonlinear vector coherent states of generalized spin-orbit Hamiltonians, J. Phys. A: Math. Theor. 42 (2009) 295202.

[8] Y. Bérubé-Lauzière, V. Hussin and L. M. Nieto, Annihilation operators and coherent states for the Jaynes-Cummings model, Phys. Rev. A 50 (1994) 1725-31.

[9] C. Brif and Y. Ben-Aryeh, Subcoherent $p$-representation for non-classical photon states, Quantum Opt. 6 (1994) 391-6.

[10] K. E. Cahill and R. J. Glauber, Density Operators and Quasiprobability Distributions, Phys. Rev. 177 (1969) 1882.

[11] M. Daoud and V. Hussin, General sets of coherent states and the Jaynes-Cummings model, J. Phys. A: Math. Gen. 35 (2002) 7381-7402.

[12] V. V. Dodonov, 'Nonclassical' states in quantum optics: a 'squeezed' review of the first 75 years, $J$. Opt. B: Quantum Semiclass. Opt. 4 (2002) R1.

[13] J. P. Gazeau, Coherent States in Quantum Physics (Wiley-VCH, Berlin, 2009).

[14] M. N. Hounkonnou and E. B. Ngompe Nkouankam, New $(p, q ; \mu, v, f)$-deformed states, J. Phys. A: Math. Theor. 40 (2007) 12113-12130.

[15] K. Husimi, Some Formal Properties of the Density Matrix, Proc. Phys. Math. Soc. Jpn 22 (1940) 264.

[16] A. Jannussis, G. Brodimas, D. Sourlas and V. Zisis, Remarks on the q-quantization, Lett. Nuovo Cimento 30 (1981) 123; G. Brodimas, A. Jannussis, D. Sourlas, V. Zisis and P. Poulopoulos, Para-Bose Operators, Lett. Nuovo Cimento 31 (1981) 177; A. Jannussis, G. Brodimas and R. Mignani, Quantum groups and Lie-admissible time evolution, J. Phys. A: Math. Gen. 24 (1991) L775; G. Brodimas, A. Jannussis and R. Mignani, Bose realization of a non-canonical Heisenberg algebra, J. Phys. A: Math. Gen. 25 (1992) L329.

[17] E. T. Jaynes and F. W. Cummings, Comparison of Quantum and Semiclassical Radiation Theory with Application to the Beam Maser, Proc. IEEE 51 (1963) 89.

[18] X.-M. Liu and B. Li, Even and odd nonlinear charge coherent states and their nonclassical properties, J. Phys. A: Math. Theor. 45 (2012) 415307.

[19] L. Mandel and E. Wolf, Optical coherence and quantum optics (Cambridge University Press, Cambridge, 1995).

[20] V. I. Man'ko, G. Marmo, S. Solimeno and F. Zaccaria, Physical nonlinear aspects of classical and quantum q-oscillators, Int. J. Mod. Phys. A 8 (1993) 3577; Correlation functions of quantum q-oscillators, Phys. Lett. A 176 (1993) 173; V. I. Man'ko, G. Marmo, E. C. G. Sudarshan and F. Zaccaria, fOscillators, Proceedings of the IY Wigner Symposium, Guadalajara, Mexico, August 1995 (World Scientific, Singapore, in press); V. I. Man'ko, G. Marmo, E. C. G. Sudarshan and F. Zaccaria, f-Oscillators and nonlinear coherent states, Phys. Scr. 55 (1997) 528-541.

[21] R. L. de Matos Filho and W. Vogel, Nonlinear coherent states, Phys. Rev. A 54 (1996) 4560.

[22] R. S. Meltzer and K. S. Hong, Electron-phonon interactions in insulating nanoparticles: $\mathrm{Eu}_{2} \mathrm{O}_{3}, \mathrm{Phys}$. Rev. B 61 (2000) 3396.

[23] T. Müller, T. Moldaschl, W. Parz, S. Golka, G. Strasser and K. Unterrainer, Femtosecond Spectral Hole Burning Spectroscopy as a Probe of Exciton Dynamics in Quantum Dots, Acta Phys. Polonica A 113 (2008) 777.

[24] R. Parthasarathy and R. Sridhar, A diagonal representation of the quantum density matrix using $q$-boson oscillator coherent states, Phys. Lett. A 305 (2002) 105-110.

[25] K. A. Penson and A. I. Solomon, New generalized coherent states, J. Math. Phys. 40 (1999) 2354.

[26] A. M. Perelomov, Generalized Coherent States and Their Applications (Berlin, Springer, 1996).

[27] C. Quesne, New $q$-deformed coherent states with an explicitly known resolution of unity, J. Phys. A: Math. Gen. 35 (2002) 9213; 
C. Quesne, K. A. Penson and V. M. Tkachuk, Geometrical and physical properties of maths-type $q$ deformed coherent states for $0<q<1$ or $q>1$, Phys. Lett. A 313 (2003) 29.

[28] B. Roy and P. Roy, New nonlinear coherent states and some of their nonclassical properties, J. Opt. B: Quantum Semiclass. Opt. 2 (2000) 65-68.

[29] E. Schrödinger, Der stetige Übergang von der Mikro-zur Makromechanik, Naturwiss. 14 (1926) 664.

[30] D. T. Simon and M. R. Geller, Electron-phonon dynamics in an ensemble of nearly isolated nanoparticles, Phys. Rev. B 64 (2001) 224504.

[31] S. E. Ulloa and M. Zarea, Landau level mixing by full spin-orbit interactions, Phys. Rev. B 72 (2005) 085342.

[32] H. S. Yang, M. R. Geller and W. M. Dennis, New Mechanism for Electronic Energy Relaxation in Nanocrystals, preprint (2000), arXiv:cond-mat/0008221.

[33] H. S. Yang, K. S. Hong, S. P. Feofilov, B. M. Tissue, R. S. Meltzer and W. M. Dennis, One phonon relaxation processes in $\mathrm{Y}_{2} \mathrm{O}_{3}: \mathrm{Eu}^{3+}$ nanocrystals, Physica $\mathrm{B} \mathbf{2 6 3}$ (1999) 476.

[34] W-M. Zhang, D. H. Feng and R. Gilmore, Coherent states: Theory and some applications, Rev. Mod. Phys. 62 (1990) 867.

\section{Appendix A. Proof of the Proposition 4.1}

(i) Hermiticity

From (4.1), we get

$$
\mathscr{K}\left(\mathbf{z}^{\prime}, \mathbf{z}\right)=\left\langle\eta_{\mathbf{z}} \mid \eta_{\mathbf{z}^{\prime}}\right\rangle=\frac{\mathscr{N}\left(\overline{\mathbf{z}} \mathbf{z}^{\prime}\right)}{\sqrt{\mathscr{N}\left(\left|\mathbf{z}^{\prime}\right|^{2}\right) \mathscr{N}\left(|\mathbf{z}|^{2}\right)}}
$$

and thereby

$$
\begin{aligned}
\overline{\mathscr{K}\left(\mathbf{z}^{\prime}, \mathbf{z}\right)} & =\overline{\left\langle\eta_{\mathbf{z}} \mid \eta_{\mathbf{z}^{\prime}}\right\rangle} \\
& =\frac{\mathscr{N}\left(\overline{\left.\mathbf{z}^{\prime} \mathbf{z}\right)}\right.}{\sqrt{\mathscr{N}\left(\left|\mathbf{z}^{\prime}\right|^{2}\right) \mathscr{N}\left(|\mathbf{z}|^{2}\right)}}=\mathscr{K}\left(\mathbf{z}, \mathbf{z}^{\prime}\right) .
\end{aligned}
$$

(ii) Positivity

Using again (4.1), we get

$$
\begin{aligned}
\mathscr{K}(\mathbf{z}, \mathbf{z})=\left\langle\eta_{\mathbf{z}} \mid \eta_{\mathbf{z}}\right\rangle & =\frac{\mathscr{N}(\overline{\mathbf{z}} \mathbf{z})}{\sqrt{\mathscr{N}\left(|\mathbf{z}|^{2}\right) \mathscr{N}\left(|\mathbf{z}|^{2}\right)}} \\
& =\frac{\mathscr{N}\left(|\mathbf{z}|^{2}\right)}{\mathscr{N}\left(|\mathbf{z}|^{2}\right)}=1>0
\end{aligned}
$$

implying that $\mathscr{K}(\mathbf{z}, \mathbf{z})>0$.

(iii) Idempotence

The left-hand side of the expression (4.4) can be written, by use of the definition (4.1), as

$$
\begin{aligned}
\int_{\mathscr{D}} d \mu\left(\mathbf{z}^{\prime \prime}, \overline{\mathbf{z}^{\prime \prime}}\right) \mathscr{K}\left(\mathbf{z}, \mathbf{z}^{\prime \prime}\right) \mathscr{K}\left(\mathbf{z}^{\prime \prime}, \mathbf{z}^{\prime}\right)= & \int_{\mathscr{D}} d \mu\left(\mathbf{z}^{\prime \prime}, \overline{\mathbf{z}^{\prime \prime}}\right)\left\{\frac{\mathscr{N}\left(\overline{\left.\mathbf{z}^{\prime \prime} \mathbf{z}\right)}\right.}{\sqrt{\mathscr{N}\left(|\mathbf{z}|^{2}\right) \mathscr{N}\left(\left|\mathbf{z}^{\prime \prime}\right|^{2}\right)}}\right\} \\
& \times\left\{\frac{\mathscr{N}\left(\overline{\left.\mathbf{z}^{\prime} \mathbf{z}^{\prime \prime}\right)}\right.}{\sqrt{\mathscr{N}\left(\left|\mathbf{z}^{\prime}\right|^{2}\right) \mathscr{N}\left(\left|\mathbf{z}^{\prime \prime}\right|^{2}\right)}}\right\}
\end{aligned}
$$




$$
\begin{aligned}
= & \left\{\frac{1}{\sqrt{\mathscr{N}\left(|\mathbf{z}|^{2}\right) \mathscr{N}\left(\left|\mathbf{z}^{\prime}\right|^{2}\right)}} \sum_{\mathbf{n}=0}^{\infty} \frac{\overline{\mathbf{z}^{\prime}}}{\left\{\mathbf{n} \mathbf{z}^{\mathbf{n}}\right\} !}\right\} \\
& \times \int_{\mathscr{D}} d \mu\left(\mathbf{z}^{\prime \prime}, \overline{\left.\mathbf{z}^{\prime \prime}\right)} \frac{1}{\mathscr{N}\left(\left|\mathbf{z}^{\prime \prime}\right|^{2}\right)}\left\{\sum_{\mathbf{n}=0}^{\infty} \frac{\left|\mathbf{z}^{\prime \prime}\right|^{2}}{\{\mathbf{n}\} !}\right\}\right. \\
\int_{\mathscr{D}} d \mu\left(\mathbf{z}^{\prime \prime}, \overline{\mathbf{z}^{\prime \prime}}\right) \mathscr{K}\left(\mathbf{z}, \mathbf{z}^{\prime \prime}\right) \mathscr{K}\left(\mathbf{z}^{\prime \prime}, \mathbf{z}^{\prime}\right)= & \frac{1}{\sqrt{\mathscr{N}\left(|\mathbf{z}|^{2}\right) \mathscr{N}\left(\left|\mathbf{z}^{\prime}\right|^{2}\right)}} \sum_{\mathbf{n}=0}^{\infty} \frac{\mathbf{z}^{\prime} \mathbf{z}^{\mathbf{n}}}{\{\mathbf{n}\} !} \\
= & : \mathscr{K}\left(\mathbf{z}, \mathbf{z}^{\prime}\right)
\end{aligned}
$$

where the following relations

$$
\frac{1}{\mathscr{N}\left(|\mathbf{z}|^{2}\right)} \sum_{\mathbf{n}=0}^{\infty} \frac{|\mathbf{z}|^{2}}{\{\mathbf{n}\} !}=1, \quad \int_{\mathscr{D}} d \mu(\mathbf{z}, \overline{\mathbf{z}})=1
$$

are used.

\section{Appendix B. Proof of the Proposition 5.1}

(i) Hermiticity

We have

$$
\rho_{[\mathbf{k}]}\left(\mathbf{z}, \mathbf{z}^{\prime}\right)=\left\langle\eta_{\mathbf{z}}\left|\rho_{[\mathbf{k}]}\right| \eta_{\mathbf{z}^{\prime}}\right\rangle
$$

and thereby

$$
\begin{aligned}
& \overline{\rho_{[\mathbf{k}]}\left(\mathbf{z}, \mathbf{z}^{\prime}\right)}=\overline{\left\langle\eta_{\mathbf{z}}\left|\rho_{[\mathbf{k}]}\right| \eta_{\mathbf{z}^{\prime}}\right\rangle} \\
& =\left\langle\eta_{\mathbf{z}^{\prime}}\left|\rho_{[\mathbf{k}]}\right| \eta_{\mathbf{z}}\right\rangle=\rho_{[\mathbf{k}]}\left(\mathbf{z}^{\prime}, \mathbf{z}\right) .
\end{aligned}
$$

(ii) Positivity

From (5.15) and (5.30), we get

$$
\begin{aligned}
\left\langle\eta_{\mathbf{z}}\left|\rho_{[\mathbf{k}]}\right| \eta_{\mathbf{z}}\right\rangle & =\left\langle\eta_{\mathbf{z}}\left|\rho_{[\mathbf{k}]} \int_{\mathscr{D}} d \mu(\mathbf{z}, \overline{\mathbf{z}}) P\left(|\mathbf{z}|^{2}\right)\right| \eta_{\mathbf{z}}\right\rangle \\
& =\sum_{\mathbf{m}, \mathbf{n}=0}^{\infty} \int_{\mathscr{D}} d \mu(\mathbf{z}, \overline{\mathbf{z}})\left(\mathscr{N}\left(|\mathbf{z}|^{2}\right)\right)^{-1} P\left(|\mathbf{z}|^{2}\right) \rho_{[\mathbf{k}]}(\mathbf{n}, \mathbf{m}) \frac{r^{\mathbf{m}+\mathbf{n}} e^{-l(\mathbf{n}-\mathbf{m}) \theta}}{\sqrt{\{\mathbf{n}\} !} \sqrt{\{\mathbf{m}\} !}} \\
& =\sum_{\mathbf{n}=0}^{\infty} \rho_{[\mathbf{k}]}(\mathbf{n}, \mathbf{n})\left\{\int_{\mathscr{D}} d \mu(\mathbf{z}, \overline{\mathbf{z}}) P\left(|\mathbf{z}|^{2}\right)\left(\mathscr{N}\left(|\mathbf{z}|^{2}\right)\right)^{-1} \frac{|\mathbf{z}|^{2 \mathbf{n}}}{\{\mathbf{n}\} !}\right\} \\
& =\sum_{\mathbf{n}=0}^{\infty}\left[\rho_{[\mathbf{k}]}(\mathbf{n}, \mathbf{n})\right]^{2} \\
& =\sum_{\mathbf{n}=0}^{\infty}\left\{2 \pi \int_{0}^{L} \frac{r^{2 \mathbf{n}}}{\{\mathbf{n}\} !} \frac{P\left(r^{2}\right)}{\mathscr{N}\left(r^{2}\right)} d \lambda(r)\right\}^{2}>0
\end{aligned}
$$

implying that

$$
\left\langle\eta_{\mathbf{z}}\left|\rho_{[\mathbf{k}]}\right| \eta_{\mathbf{z}}\right\rangle=\rho_{[\mathbf{k}]}(\mathbf{z}, \mathbf{z})>0 .
$$


(iii) Idempotence

By setting $d \mu\left(\mathbf{z}^{\prime \prime}, \overline{\mathbf{z}^{\prime \prime}}\right)=\mathscr{N}\left(\left|\mathbf{z}^{\prime \prime}\right|^{2}\right) d \lambda\left(r^{\prime \prime}\right) d \theta$ and using the relation $\sum_{\mathbf{n}=0}^{\infty} \rho_{[\mathbf{k}]}(\mathbf{n}, \mathbf{n})=$ 1 , we get from (5.12)

$$
\begin{aligned}
& \int_{\mathscr{D}} d \mu\left(\mathbf{z}^{\prime \prime}, \overline{\mathbf{z}}^{\prime \prime}\right) \rho_{[\mathbf{k}]}\left(\mathbf{z}, \mathbf{z}^{\prime \prime}\right) \rho_{[\mathbf{k}]}\left(\mathbf{z}^{\prime \prime}, \mathbf{z}^{\prime}\right) \\
& =\int_{\mathscr{D}} d \mu\left(\mathbf{z}^{\prime \prime}, \overline{\mathbf{z}^{\prime \prime}}\right)\left[\left[\mathscr{N}\left(|\mathbf{z}|^{2}\right) \mathscr{N}\left(\left|\mathbf{z}^{\prime \prime}\right|^{2}\right)\right]^{-1 / 2} \sum_{\mathbf{n}, \mathbf{m}=0}^{\infty} \rho_{[\mathbf{k}]}(\mathbf{n}, \mathbf{m}) \frac{\overline{\mathbf{z}}^{\mathbf{n}}}{\sqrt{\{\mathbf{n}\} !}} \frac{\mathbf{z}^{\prime \prime \mathbf{m}}}{\sqrt{\{\mathbf{m}\} !}}\right] \\
& \times\left[\left[\mathscr{N}\left(\left|\mathbf{z}^{\prime \prime}\right|^{2}\right) \mathscr{N}\left(\left|\mathbf{z}^{\prime}\right|^{2}\right)\right]^{-1 / 2} \sum_{\mathbf{n}, \mathbf{m}=0}^{\infty} \rho_{[\mathbf{k}]}(\mathbf{n}, \mathbf{m}) \frac{\overline{\mathbf{z}}^{\prime \prime} \mathbf{n}}{\sqrt{\{\mathbf{n}\} !}} \frac{\mathbf{z}^{\prime \mathbf{m}}}{\sqrt{\{\mathbf{m}\} !}}\right] \\
& =\left[\left[\mathscr{N}\left(|\mathbf{z}|^{2}\right) \mathscr{N}\left(\left|\mathbf{z}^{\prime}\right|^{2}\right)\right]^{-1 / 2} \sum_{\mathbf{n}, \mathbf{m}=0}^{\infty} \rho_{[\mathbf{k}]}(\mathbf{n}, \mathbf{m}) \frac{\overline{\mathbf{z}}^{\mathbf{n}}}{\sqrt{\{\mathbf{n}\} !}} \frac{\mathbf{z}^{\prime \mathbf{m}}}{\sqrt{\{\mathbf{m}\} !}}\right] \\
& \times\left[\sum_{\mathbf{n}=0}^{\infty} \rho_{[\mathbf{k}]}(\mathbf{n}, \mathbf{n})\right] \\
& =\rho_{[\mathbf{k}]}\left(\mathbf{z}, \mathbf{z}^{\prime}\right) .
\end{aligned}
$$

